\title{
Motivic invariants of arc-symmetric sets and blow-Nash equivalence
}

\author{
Goulwen Fichou
}

\begin{abstract}
We define invariants of the blow-Nash equivalence of Nash function germs, in a similar way to the motivic zeta functions of Denef and Loeser. As a key ingredient, we extend the virtual Betti numbers, which were known for real algebraic sets, to a generalized Euler characteristic for projective constructible arc-symmetric sets. Actually we prove more: the virtual Betti numbers are not only algebraic invariants, but also Nash invariants of arcsymmetric sets. Our zeta functions enable one to distinguish the blow-Nash equivalence classes of Brieskorn polynomials of two variables. We prove moreover that there are no moduli for the blow-Nash equivalence in the case of an algebraic family with isolated singularities.
\end{abstract}

\section{Introduction}

In the study of real analytic function germs, the choice of a good equivalence relation between germs is a crucial topic. Whereas the topological equivalence is too coarse and the $C^{1}$-equivalence too fine, the blow-analytic equivalence, a notion introduced by T.-C. Kuo in 1985 (see [Kuo85], and [FKK98] for a survey) seems to behave better, especially with respect to finiteness properties. In this paper, we will focus on a particular case of blow-analytic equivalence, called blow-Nash equivalence, for which we add algebraicity assumptions.

Let $f, g:\left(\mathbb{R}^{d}, 0\right) \longrightarrow(\mathbb{R}, 0)$ be Nash function germs. Then $f$ and $g$ are said to be blow-Nash equivalent if there exist two algebraic modifications

$$
\pi_{f}:\left(M_{f}, \pi_{f}^{-1}(0)\right) \longrightarrow\left(\mathbb{R}^{d}, 0\right) \text { and } \pi_{g}:\left(M_{g}, \pi_{g}^{-1}(0)\right) \longrightarrow\left(\mathbb{R}^{d}, 0\right),
$$

and a Nash isomorphism $\phi:\left(M_{f}, \pi_{f}^{-1}(0)\right) \longrightarrow\left(M_{g}, \pi_{g}^{-1}(0)\right)$, that is an analytic isomorphism with semi-algebraic graph, which respects the multiplicity of the jacobian determinants of $\pi_{f}$ and $\pi_{g}$ and which induces a homeomorphism $h$ between neighbourhoods of 0 in $\mathbb{R}^{d}$ such that $f=g \circ h$. Here, by a modification $\pi$ of $f$, we mean a proper birational map which is an isomorphism over the complement of the zero locus of $f$ and such that $f \circ \pi$ and jac $\pi$ have simultaneously only normal crossings. One can define such a relation on Nash sets, and Koike [Koi97, Koi00] proved that finiteness properties hold in this setting. In the case of germs of functions, the question of moduli is still open in general. However, in $\S 4$, we prove that there are no moduli for a Nash family with isolated singularities under some algebraic assumptions on the modifications. In particular, an algebraic family of isolated singularities does not admit moduli for the blow-Nash equivalence.

A common issue for blow-analytic equivalence and blow-Nash equivalence is to prove that, when it is the case, two given germs of real analytic functions are not equivalent. The difficulty arises in the lack of invariants known for these relations. Up to now, just two kinds of invariants

Received 9 October 2003, accepted in final form 31 May 2004, published online 21 April 2005.

2000 Mathematics Subject Classification 14B05, 14P20, 14P25, 32S15.

Keywords: arc-symmetric sets, motivic integration, singularities of Nash function germs, blow-Nash equivalence.

This journal is (C) Foundation Compositio Mathematica 2005. 


\section{G. FICHOU}

have been known: the Fukui invariants and the zeta functions of Koike and Parusiński [KP03]. With an analytic function germ $f$, the Fukui invariants associate the set of possible orders $n$ of series $f \circ \gamma(t)=a_{n} t^{n}+\cdots, a_{n} \neq 0$, for $\gamma:(\mathbb{R}, 0) \longrightarrow\left(\mathbb{R}^{d}, 0\right)$ an analytic arc [Fuk97, IKK02]. There exists also a version of the Fukui invariants related to the sign of $f$. Using motivic integration combined with the construction of a computable motivic invariant for arc-symmetric sets, the virtual Betti numbers, we introduce in this paper zeta functions $Z(T)$ and $Z^{ \pm}(T)$ of a real analytic function germ that belong to $\mathbb{Z}\left[u, u^{-1}\right][[T]]$, and take into account not only the orders of the series $f \circ \gamma(t)$ but also the geometry of the sets $\mathcal{X}_{n}(f)$ of $\operatorname{arcs} \gamma$ that realize a given order $n$ (for precise definitions, see $\S 3.1$ ). These zeta functions are similar to the motivic zeta functions of Denef and Loeser [DL01], and also to those of Koike and Parusiński, defined using the Euler characteristic with compact supports.

We prove that our zeta functions are invariants of the blow-Nash equivalence. The proof is directly inspired by the work of Denef and Loeser via their formulae for the zeta functions in terms of a modification of the zero locus of the given function germ (Propositions 3.2 and 3.5). It uses the powerful machinery of motivic integration, a theory introduced by Kontsevich in 1995 [Kon95] and developed further by Denef and Loeser [DL98, DL99, DL01, DL02], in particular the fundamental change of variables formula (Proposition 3.10).

In order to dispose of computable invariants, motivic integration requires computable measures, or in other words generalized Euler characteristics. A generalized Euler characteristic is an additive and multiplicative invariant defined on the level of the Grothendieck group of varieties. In our setting of the blow-Nash equivalence, we need invariants of the Zariski constructible sets over real algebraic varieties $\mathcal{X}_{n}(f)$ (real algebraic variety in the sense of [BCR98]), and we ask them to be respected by Nash isomorphisms. This leads naturally to the category of Nash varieties, and more generally of arc-symmetric sets, a category introduced by Kurdyka [Kur88].

In $\S 2$, we give conditions, inspired by a result of Bittner [Bit04], on an invariant defined on connected components of compact non-singular real algebraic varieties such that it extends to an additive invariant on the constructible category of arc-symmetric sets. Additive means that $\chi(A)=$ $\chi(B)+\chi(A \backslash B)$ for a closed inclusion $B \subset A$ of arc-symmetric sets. As a fundamental example, we prove that the Betti number $b_{k}$ with $\mathbb{Z}_{2}$ coefficients defined on connected components $A$ of compact non-singular real algebraic sets by $b_{k}(A)=\operatorname{dim} H_{k}\left(A, \mathbb{Z}_{2}\right)$ give such an additive invariant $\beta_{k}$ on arc-symmetric sets for each $k \in \mathbb{N}$ (Corollary 2.5), called a $k$-virtual Betti number. We make them

multiplicative by putting $\beta(A)=\sum_{k=0}^{\operatorname{dim} A} \beta_{k}(A) u^{k} \in \mathbb{Z}[u]$, called the virtual Poincaré polynomial of $A$.

Let us emphasize the fact that the unique such additive invariant known up to now in the real case is the classical Euler characteristic with compact supports, and in fact it is the unique generalized Euler characteristic for semi-algebraic sets up to homeomorphism [Qua01].

The virtual Poincaré polynomial is not a topological invariant. It respects the dimension (see Remark 2.11) whereas the Euler characteristic with compact supports may identify the dimension. Note that the virtual Betti numbers have been proven recently to be additive invariants of real algebraic varieties by McCrory and Parusiński in [MCP03] and independently by Totaro [Tot02]; in this paper, we extend these numbers to the more general context of arc-symmetric sets, and we prove the invariance not only under algebraic isomorphisms but also under Nash isomorphisms (see Definition 2.15).

\section{Arc-symmetric sets}

The category of arc-symmetric sets contains the real algebraic varieties and, in some sense, this category has a better behaviour than that of real algebraic varieties, similar to complex algebraic varieties. For example, a closed and irreducible arc-symmetric set is connected whereas a closed and irreducible real algebraic variety may have as many connected components as one wants! 


\section{MOTIVIC INVARIANTS OF BLOW-NASH EQUIVALENCE}

In this section, we introduce arc-symmetric sets, with a definition slightly different from that of Kurdyka, and we state their basic properties in relation to closure, dimension and irreducibility. Then, we study in more detail the non-singular arc-symmetric sets and especially the resolution of singularities for arc-symmetric sets.

\subsection{Arc-symmetric sets and closure}

We fix a compactification of $\mathbb{R}^{n}$, for instance $\mathbb{R}^{n} \subset \mathbb{P}^{n}$.

Definition 1.1. Let $A \subset \mathbb{P}^{n}$ be a semi-algebraic set. We say that $A$ is arc-symmetric if, for every real analytic arc $\gamma:]-1,1\left[\longrightarrow \mathbb{P}^{n}\right.$ such that $\gamma(]-1,0[) \subset A$, there exists $\epsilon>0$ such that $\gamma(] 0, \epsilon[) \subset A$.

This definition is due to Parusiński [Par04]. Note that a closed arc-symmetric set is necessarily compact. This definition differs from that of Kurdyka [Kur88], who considers only closed arc-symmetric sets in $\mathbb{R}^{n}$. One can think of our arc-symmetric sets as being the projective constructible arc-symmetric sets of Kurdyka. We remark moreover that an arc-symmetric set need not be an analytic variety (cf. [Kur88, Example 1.2]).

\section{Example 1.2.}

(i) A real algebraic variety is an arc-symmetric set. A connected component of a compact real algebraic variety is also an arc-symmetric set.

(ii) The two-dimensional sheet of the Whitney umbrella of the equation $z x^{2}=y^{2}$ is arc-symmetric.

We remark that the arc-symmetric sets form a constructible category of semi-algebraic sets in the sense of [Par04], denoted $\mathcal{A S}$, that is:

(a) $\mathcal{A S}$ contains the algebraic sets;

(b) $\mathcal{A S}$ is stable under set-theoretic operations $\cup, \cap, \backslash$;

(c) $\mathcal{A S}$ is stable by inverse images of $\mathcal{A S}$-maps (i.e. whose graph is in $\mathcal{A S}$ ) and by images of injective $\mathcal{A S}$-maps;

(d) each $A \in \mathcal{A S}$ has a well-defined fundamental class with coefficients in $\mathbb{Z}_{2}$.

In particular there is a notion of closure in $\mathcal{A S}$ (we refer to [Par04] for a proof).

Proposition 1.3. Every $A \in \mathcal{A S}$ admits a smallest arc-symmetric set, denoted by $\bar{A}^{\mathcal{A S}}$, containing $A$ and closed in $\mathbb{P}^{n}$.

Remark 1.4. Arc-symmetric sets are not stable under the euclidean closure. Consider for example the regular part $A$ of the Whitney umbrella $z x^{2}=y^{2}$. The closure of $A$ in $\mathcal{A S}$ is the entire Whitney umbrella.

We can define irreducible arc-symmetric sets in the usual way: $A \in \mathcal{A S}$ is irreducible if the existence of a decomposition $A=B \cup C$, with $B$ and $C$ closed in $A$ and arc-symmetric, implies that either $B \subset C$ or $C \subset B$. We remark that an irreducible arc-symmetric set is not necessarily connected, with our definition of arc-symmetric sets (as an example, consider a hyperbola in the plane). Nevertheless, as proved in [Kur88], an arc-symmetric set $A$ admits a unique decomposition into a finite union of irreducible arc-symmetric sets closed in $A$.

Proposition 1.5. If $A \in \mathcal{A S}$ is irreducible, then so is $\bar{A}^{\mathcal{A S}}$.

Proof. Assume that $\bar{A}^{\mathcal{A S}}$ can be decomposed into $\bar{A}^{\mathcal{A S}}=B \cup C$ with $B$ and $C$ arc-symmetric and closed in $\bar{A}^{\mathcal{A S}}$. Then $B$ and $C$ are closed and $A$ splits into

$$
A=A \cap \bar{A}^{\mathcal{A S}}=(A \cap B) \cup(A \cap C),
$$




\section{G. FICHOU}

with $A \cap B$ and $A \cap C$ arc-symmetric and closed in $A$. But $A$ is irreducible so either $A \cap B \subset A \cap C$ or the reverse inclusion holds. By symmetry, one can assume that $A \cap B \subset A \cap C$. Then $A$ equals $A \cap C$, so $A$ is included in $C$ and finally $\bar{A}^{\mathcal{A S}}$ is equal to $C$ because $C$ is arc-symmetric and closed.

Define the dimension of an arc-symmetric set to be its dimension as a semi-algebraic set. Then, it equals the dimension of its Zariski closure in the projective space [Kur88] (recall that we consider real algebraic varieties in the sense of [BCR98]) and therefore

$$
\operatorname{dim} A=\operatorname{dim} \bar{A}^{\mathcal{A S}}=\operatorname{dim} \bar{A}^{Z}
$$

for $A \in \mathcal{A S}$. The following result relates the dimension of an arc-symmetric set to the dimension of its arc-symmetric closure. It will be useful in the sequel when dealing with proofs by induction, e.g. for Theorems 2.3 and 2.16 .

Proposition 1.6. Let $A \in \mathcal{A S}$. Then $\bar{A}^{\mathcal{A S}}=A \cup \overline{\bar{A} \backslash A}^{\mathcal{A S}}$. In particular $\operatorname{dim} \bar{A}^{\mathcal{A S}} \backslash A<\operatorname{dim} A$.

Proof. Note that, as a union of arc-symmetric sets, $F=A \cup \overline{\bar{A} \backslash A}^{\mathcal{A S}}$ is arc-symmetric. Moreover $F$ can be decomposed into

$$
F=A \cup(\bar{A} \backslash A) \cup\left(\overline{\bar{A} \backslash A}^{\mathcal{A S}}\right)=\bar{A} \cup \overline{\bar{A} \backslash A}^{\mathcal{A S}},
$$

and thus $F$ is closed. So the inclusion $\bar{A}^{\mathcal{A S}} \subset F$ holds.

Moreover $\bar{A}$ is included in $\bar{A}^{\mathcal{A S}}$ because $\bar{A}^{\mathcal{A S}}$ is closed. Thus $\overline{\bar{A} \backslash A}^{\mathcal{A S}} \subset \bar{A}^{\mathcal{A S}}$, and so $F \subset \bar{A}^{\mathcal{A S}}$. Consequently $F=\bar{A}^{\mathcal{A S}}$.

We can adapt Proposition 1.5 of [Kur99] to our definition of arc-symmetric sets. It is another example of the good behaviour of irreducible arc-symmetric sets.

Proposition 1.7. Let $A \in \mathcal{A S}$ be irreducible, and $B \subset A$ be a closed arc-symmetric subset of $A$ of the same dimension. Then $B=A$.

Proof. $A$ can be decomposed into the union of two arc-symmetric sets closed in $A$ as follows: $A=B \cup\left(\overline{A \backslash B}^{\mathcal{A S}} \cap A\right)$. Then, by irreducibility of $A$, either $B \subset \overline{A \backslash B}^{\mathcal{A S}} \cap A$ or $\overline{A \backslash B}^{\mathcal{A S}} \cap A \subset B$.

In the second case $B$ is equal to $A$, and in the first one $B$ is included in $\left(\overline{A \backslash B}^{\mathcal{A S}}\right) \backslash(A \backslash B)$. But this cannot happen, for the dimension of this arc-symmetric set is strictly less than $\operatorname{dim} B$ by Proposition 1.6.

\subsection{Nonsingular arc-symmetric sets}

Let us define a non-singular arc-symmetric set with relation to its Zariski closure in the projective space.

Definition 1.8. An arc-symmetric set $A$ is non-singular if $A \cap \operatorname{Sing}\left(\bar{A}^{Z}\right)=\emptyset$.

Lemma 1.9 [Kur99]. A non-singular and connected arc-symmetric set is irreducible.

Let us state a definition of an isomorphism between arc-symmetric sets.

Definition 1.10. Let $A, B \in \mathcal{A S}$. Then $A$ is isomorphic to $B$ if and only if there exist Zariski open subsets $U$ and $V$ in $\bar{A}^{\mathcal{Z}}$ and $\bar{B}^{\mathcal{Z}}$ containing $A$ and $B$ respectively, and an algebraic isomorphism $\phi: U \longrightarrow V$ such that $\phi(A)=B$. 


\section{Motivic InVARIANTS OF BLOW-NASH EQUivalence}

Remark 1.11. At this point, we are only interested in the algebraic point of view, because we have in mind to study algebraic singularities of arc-symmetric sets, and to use Hironaka's desingularization theorem. In $\S 2.2$ we will give another definition of an isomorphism between arc-symmetric sets (see Definition 2.15).

Proposition 1.12. Let $A \in \mathcal{A S}$ be compact and non-singular. Then $A$ is isomorphic to a union of connected components of some compact non-singular real algebraic variety.

Proof. Let $X=\bar{A}^{\mathcal{Z}}$ be the Zariski closure of $A$ in the projective space, and let $\pi: \widetilde{X} \longrightarrow X$ be a resolution of the singularities of $X$. We remark that the three spaces $A, X$ and $\widetilde{X}$ have the same dimension and that $A$ is isomorphic to the subset $\pi^{-1}(A)=\widetilde{A}$ of $\widetilde{X}$ because $A \subset \operatorname{Reg}(X)$ and $\operatorname{Reg}(X)$ is a Zariski open subset of $X$ isomorphic to $\pi^{-1}(\operatorname{Reg}(X)) \subset \widetilde{X}$.

Now, denote by $\widetilde{X}=\bigcup_{i \in I} C_{i}$ the decomposition of $\widetilde{X}$ into connected components. Each $C_{i}$, $i \in I$, is a connected and non-singular arc-symmetric set of dimension $\operatorname{dim} X$, hence irreducible by Lemma 1.9. Therefore $\widetilde{A} \cap C_{i}$, whose dimension equals $\operatorname{dim} C_{i}$ by the non-singularity of $\widetilde{A}$, is either equal to $C_{i}$ or empty because of Proposition 1.7 , and so $\widetilde{A}$ is a union of connected components of $\widetilde{X}$ as claimed.

\subsection{Arc-symmetric sets and resolution of singularities}

The following proposition is an adaptation of Theorem 2.6 of [Kur88] to our definition of arcsymmetric sets. It asserts that, up to desingularization, we can think of an irreducible arc-symmetric set as being a connected component of a real algebraic variety.

Proposition 1.13. Let $A \in \mathcal{A S}$ be irreducible. Let $X$ be a compact real algebraic variety containing $A$ with $\operatorname{dim} X=\operatorname{dim} A$, and $\pi: \widetilde{X} \longrightarrow X$ a resolution of singularities for $X$ (cf. [Hir64]). Then, there exists a unique connected component $\widetilde{A}$ of $\widetilde{X}$ such that $\pi(\widetilde{A})=\overline{\operatorname{Reg}(A)}$.

Proof. Let $\widetilde{A_{0}}$ be an irreducible arc-symmetric component of dimension $\operatorname{dim} A$ of $\pi^{-1}(A)$. Such an $\widetilde{A_{0}}$ exists because the dimensions of $\pi^{-1}(A)$ and $A$ coincide. Then $\widetilde{A_{0}}$ is contained in some connected component $\widetilde{A}$ of $\widetilde{X}$. Actually $\overline{\widetilde{A}_{0}}$ AS is irreducible (because so is $\widetilde{A_{0}}$, cf. Proposition 1.5) and closed, and therefore it is connected by Proposition 1.12. Now ${\widetilde{\widetilde{A_{0}}}}^{\mathcal{A S}}$ is included in some connected component of $\widetilde{X}$, and is equal to this component by Proposition 1.7 . We can put $\widetilde{A}=\overline{\widetilde{A}_{0}} \mathcal{A S}$. Note moreover that the euclidean closure $\overline{\widetilde{A_{0}}}$ of $\widetilde{A_{0}}$ also equals $\widetilde{A}$ by the non-singularity of $\widetilde{A}$.

Let us prove that the announced equality $\pi(\widetilde{A})=\overline{\operatorname{Reg}(A)}$ holds. In fact, it suffices to prove that

$$
\operatorname{dim} \overline{\pi(\widetilde{A})}^{\mathcal{A S}} \backslash \pi(\widetilde{A})<\operatorname{dim} A,
$$

which will be done in the next lemma.

This is sufficient for the following reasons. Note first that $\overline{\pi(\widetilde{A})}^{\mathcal{A S}}$ is included in $\bar{A}^{\mathcal{A S}}$ because

$$
\pi(\widetilde{A})=\pi\left(\overline{\widetilde{A_{0}}}\right) \subset \overline{\pi\left(\widetilde{A_{0}}\right)} \subset \bar{A} \subset \bar{A}^{\mathcal{A S}} .
$$

Then, on the one hand, $\overline{\pi(\widetilde{A})}{ }^{\mathcal{A S}}$ is equal to $\bar{A}^{\mathcal{A S}}$ by Proposition 1.7 , so $\operatorname{dim} \bar{A}^{\mathcal{A S}} \backslash \pi(\widetilde{A})<\operatorname{dim} A$. Now $\operatorname{Reg}(A) \cap\left(\bar{A}^{\mathcal{A S}} \backslash \pi(\widetilde{A})\right)$ is an open subset of $\bar{A}^{\mathcal{A S}}$ of dimension strictly less than $\operatorname{dim} A$, so $\operatorname{Reg}(A) \cap\left(\bar{A}^{\mathcal{A S}} \backslash \pi(\widetilde{A})\right)=\emptyset$. This implies the inclusion $\operatorname{Reg}(A) \subset \pi(\widetilde{A})$.

On the other hand, if $E$ denotes the exceptional divisor of the resolution, then $\pi(\widetilde{A} \backslash E)$ is included in $\operatorname{Reg}\left(\bar{A}^{\mathcal{A S}}\right)$. However $\operatorname{Reg}\left(\bar{A}^{\mathcal{A S}}\right)$ is included in $\overline{\operatorname{Reg}(A)}$ because $\operatorname{dim} \bar{A}^{\mathcal{A S}} \backslash A<\operatorname{dim} A$ by Proposition 1.6.

Thus the inclusions $\pi(\widetilde{A} \backslash E) \subset \overline{\operatorname{Reg}(A)} \subset \pi(\widetilde{A})$ hold and give the conclusion by taking the closure. 


\section{G. FICHOU}

Lemma 1.14. Let $A$ and $\widetilde{A}$ be as in the proof of Proposition 1.13. Then

$$
\operatorname{dim} \overline{\pi(\widetilde{A})}^{\mathcal{A S}} \backslash \pi(\widetilde{A})<\operatorname{dim} A .
$$

Proof. Let us prove that the inclusion $\overline{\pi(\widetilde{A})}^{\mathcal{A S}} \subset \pi(\widetilde{A}) \cup \overline{\pi(E)}^{\mathcal{A S}}$ holds. Denote by $F$ the set $\pi(\widetilde{A}) \cup \overline{\pi(E)}^{\mathcal{A S}}$. We remark that if $F$ is closed and arc-symmetric, the lemma is proved.

As $\pi$ is proper, $\pi(\widetilde{A})$ is closed and so is $F$. Now, let $\gamma:]-\epsilon, \epsilon\left[\longrightarrow \mathbb{P}^{n}\right.$ be a real analytic arc such that int $\gamma^{-1}(F) \neq \emptyset$. Then

(a) either int $\gamma^{-1}\left(\overline{\pi(E)}^{\mathcal{A S}}\right) \neq \emptyset$ and $\gamma(]-\epsilon, \epsilon[) \subset \overline{\pi(E)}^{\mathcal{A S}}$

(b) or int $\gamma^{-1}\left(\pi(\widetilde{A}) \backslash \overline{\pi(E)}^{\mathcal{A S}}\right) \neq \emptyset$.

In the latter case, there exists a unique analytic arc $\widetilde{\gamma}:]-\epsilon, \epsilon\left[\longrightarrow \mathbb{P}^{m}\right.$ such that $\pi \circ \widetilde{\gamma}=\gamma$. One has $\operatorname{int} \widetilde{\gamma}^{-1}(\widetilde{A}) \neq \emptyset$, and therefore $\widetilde{\gamma}(]-\epsilon, \epsilon[) \subset \widetilde{A}$ because $\widetilde{A}$ is arc-symmetric. Finally $\gamma(]-\epsilon, \epsilon[) \subset \pi(\widetilde{A}) \subset F$, and thus $F$ is arc-symmetric.

Remark 1.15. Denote by $D$ the singular locus of $X$ and by $E$ the exceptional divisor of the resolution of Proposition 1.13. Then $\pi: \widetilde{A} \backslash E \longrightarrow \bar{A}^{\mathcal{A S}} \backslash D$ is an isomorphism of arc-symmetric sets (restriction of an algebraic isomorphism). If we add the assumption that $A$ is non-singular, then the conclusion of Proposition 1.13 becomes simply $\pi(\widetilde{A})=\bar{A}$. Moreover $\pi: \widetilde{A} \backslash\left(\widetilde{A} \backslash \pi^{-1}(A)\right) \longrightarrow A$ is an isomorphism between arc-symmetric sets, and $\widetilde{A}$ is close to $A$ is the sense that $\operatorname{dim} \widetilde{A} \backslash \pi^{-1}(A)<\operatorname{dim} A$.

Actually, it is easy to prove that the symmetric difference of $A$ and $\pi(\widetilde{A})$ consists of a semialgebraic set of dimension strictly less than $\operatorname{dim} A$. More precisely, we have the following proposition.

Proposition 1.16. Let $A$ and $\widetilde{A}$ be as in Proposition 1.13. Then

$$
\begin{gathered}
A \backslash(\pi(\widetilde{A}) \cap A)=\left\{x \in \operatorname{Sing}(A) ; \operatorname{dim}_{x} A<\operatorname{dim} A\right\}, \\
\pi(\widetilde{A}) \backslash(A \cap \pi(\widetilde{A}))=\left\{x \in \bar{A}^{\mathcal{A S}} \backslash A ; \operatorname{dim}_{x} \bar{A}^{\mathcal{A S}}=\operatorname{dim} A\right\} .
\end{gathered}
$$

Let us finish this section by stating the particular case of the 'blowing-up' of a real algebraic variety along a closed non-singular arc-symmetric set. By virtue of Proposition 1.12, it is just the blowing-up of a non-singular real algebraic variety along a non-singular centre.

Proposition 1.17. Let $Y \subset X$ be compact non-singular algebraic varieties such that $\operatorname{dim} Y<$ $\operatorname{dim} X$, and let $A \subset X$ be a connected component of $X$. Denote by $\pi: \widetilde{X} \longrightarrow X$ the blowing-up of $X$ along $Y$. Then $\pi$ is surjective and $\pi^{-1}(A)$ is a connected component of $\tilde{X}$.

\section{Virtual Betti numbers}

In the theory of motivic integration, generalized Euler characteristics play the role of a measure for certain subsets of the arc space of a variety. In $\S 2.1$, we give a new example of such a generalized Euler characteristic of arc-symmetric sets, constructed from the Betti numbers of compact nonsingular arc-symmetric sets.

This example, called the virtual Poincaré polynomial, was already known for real algebraic varieties; it has been introduced independently by McCrory and Parusiński [MCP03] and by Totaro [Tot02]. Here we define the virtual Poincaré polynomial for the larger category of arc-symmetric sets. The way to perform this is, following an idea of Bittner [Bit04], to extend an invariant of the compact non-singular arc-symmetric sets to the whole category of arc-symmetric sets. The key ingredients are the resolution of singularities, which enables us to define the invariant for all arcsymmetric sets, and the weak factorization theorem, which simplifies the proof of the independence of the choices we have to make. 


\section{Motivic InVARIANTS OF BLOW-NASH EQUivalence}

Moreover we prove, in $\S 2.2$, that the virtual Betti numbers are invariants of the arc-symmetric sets not only under algebraic isomorphisms, but also under Nash isomorphisms. This result will be useful when studying the blow-Nash equivalence of germs of real analytic functions in the next section.

\subsection{Generalized Euler characteristics of arc-symmetric sets}

Definition 2.1. An additive map on $\mathcal{A S}$ with values in an abelian group is a map $\chi$ defined on $\mathcal{A S}$ such that

(i) for arc-symmetric sets $A$ and $B$ that are isomorphic, $\chi(A)=\chi(B)$,

(ii) for a closed arc-symmetric subset $B$ of $A, \chi(A)=\chi(B)+\chi(A \backslash B)$.

If moreover $\chi$ takes values in a commutative ring and satisfies $\chi(A \times B)=\chi(A) \cdot \chi(B)$ for arc-symmetric sets $A$ and $B$, then we say that $\chi$ is a generalized Euler characteristic on $\mathcal{A S}$.

Remark 2.2.

(i) One can construct a universal generalized Euler characteristic with values in the Grothendieck ring of arc-symmetric sets [DL01]. But this ring is rather complicated, and we are interested in more computable invariants.

(ii) The Euler characteristic with compact supports is a generalized Euler characteristic on $\mathcal{A S}$, and maybe the simplest one. In fact, it is unique if we consider just semi-algebraic sets, with isomorphisms replaced by homeomorphisms [Qua01]. However, for complex algebraic varieties, a lot of such generalized Euler characteristics exist, for example deduced from mixed Hodge structures [DL01, Loo02].

The following theorem gives sufficiently good conditions, on an invariant $\chi$ over the closed (i.e. compact) and non-singular arc-symmetric sets, such that $\chi$ extends to an additive map on $\mathcal{A S}$. We state the theorem in terms of connected components of real algebraic varieties thanks to Proposition 1.12. The method is inspired by that of Bittner [Bit04], who proves the result for algebraic varieties over a field of characteristic zero.

THEOREM 2.3. Let $\chi$ be a map defined on connected components of compact non-singular real algebraic varieties with values in an abelian group and such that:

(P1) $\chi(\emptyset)=0$;

(P2) if $A$ and $B$ are connected components of compact non-singular real algebraic varieties that are isomorphic as arc-symmetric sets, then $\chi(A)=\chi(B)$;

(P3) with the notations and assumptions of Proposition 1.17,

$$
\chi\left(\pi^{-1}(A)\right)-\chi\left(\pi^{-1}(A) \cap \pi^{-1}(A \cap Y)\right)=\chi(A)-\chi(A \cap Y) .
$$

Then $\chi$ extends uniquely to an additive map defined on $\mathcal{A S}$.

Remark 2.4. Property (P3) of Theorem 2.3 is a kind of additivity property for non-singular arc-symmetric sets.

Before giving the proof of the theorem after Remark 2.11, let us state some consequences. First, this result enables us to give another example of such an additive map by considering the homology with coefficients in $\mathbb{Z}_{2}$. For $i \in \mathbb{N}$, denote by $b_{i}$ the $i$ th Betti number with coefficients in $\mathbb{Z}_{2}$, defined by $b_{i}(\cdot)=\operatorname{dim} H_{i}\left(\cdot, \mathbb{Z}_{2}\right)$.

Corollary 2.5. There exist additive maps on $\mathcal{A S}$ with values in $\mathbb{Z}$, denoted $\beta_{i}$ and called virtual Betti numbers, such that $\beta_{i}$ coincides with the classical Betti number $b_{i}$ on the connected components of compact non-singular real algebraic varieties. 


\section{G. FICHOU}

Remark 2.6. McCrory and Parusiński [MCP03] have proven the same result for real algebraic varieties. In particular, they have defined the virtual Betti numbers of real algebraic varieties. The proof below is inspired by their arguments.

Proof of Corollary 2.5. We adopt the notations of property (P3) of Theorem 2.3. One checks in a similar way to [MCP03] that there exist exact sequences of the form

$$
0 \longrightarrow H_{i}\left(\pi^{-1}(A) \cap \pi^{-1}(A \cap Y)\right) \longrightarrow H_{i}(A \cap Y) \oplus H_{i}\left(\pi^{-1}(A)\right) \longrightarrow H_{i}(A) \longrightarrow 0,
$$

where the homology is considered with coefficients in $\mathbb{Z}_{2}$. Therefore

$$
b_{i}\left(\pi^{-1}(A)\right)-b_{i}\left(\pi^{-1}(A) \cap \pi^{-1}(A \cap Y)\right)=b_{i}(A)-b_{i}(A \cap Y),
$$

and we can apply Theorem 2.3 .

It turns out to be easy to adapt Theorem 2.3 in order to obtain not only additive maps but also generalized Euler characteristics.

Theorem 2.7. Let $\chi$ be as in Theorem 2.3. Assume moreover that $\chi$ takes values in a commutative ring, and that for connected components of compact non-singular real algebraic varieties $A$ and $B$, the relation $\chi(A \times B)=\chi(A) \chi(B)$ holds. Then the unique extension of $\chi$ on $\mathcal{A S}$ of Theorem 2.3 is a generalized Euler characteristic.

The proof of this theorem is given at the end of this subsection. The following corollary is an immediate consequence of the Künneth formula.

Corollary 2.8. Let $\beta$ be defined by $\beta(A)=\sum_{i=0}^{\operatorname{dim} A} \beta_{i}(A) u^{i}$ for $A \in \mathcal{A S}$. Then $\beta$ is a generalized Euler characteristic on $\mathcal{A S}$, called the virtual Poincaré polynomial.

Remark 2.9 .

(i) The name 'virtual Poincaré polynomial' is inspired by [Fu193], where Fulton studies such a virtual Poincaré polynomial for complex algebraic varieties. It is related to the weighted characteristic associated with mixed Hodge structures.

(ii) By uniqueness in Theorem 2.7, one recovers the Euler characteristic with compact supports by evaluating $\beta$ at $u=-1$.

(iii) The virtual Poincaré polynomial is not a topological invariant [MCP03].

Example 2.10.

(i) If $\mathbb{P}^{k}$ denotes the real projective space of dimension $k$, which is non-singular and compact, then $\beta\left(\mathbb{P}^{k}\right)=1+u+\cdots+u^{k}$. Now, compactify the affine line $\mathbb{A}_{\mathbb{R}}^{1}$ in $\mathbb{P}^{1}$ by adding one point at infinity. By additivity $\beta\left(\mathbb{A}_{\mathbb{R}}^{1}\right)=\beta\left(\mathbb{P}^{1}\right)-\beta$ (point $)=u$, and so $\beta\left(\mathbb{A}_{\mathbb{R}}^{k}\right)=u^{k}$.

(ii) Let $W$ be the Whitney umbrella, and $L$ be the line included in $W$. Then the additivity property implies $\beta(W)=\beta(W \backslash L)+\beta(L)$. Moreover $W \backslash L$ is isomorphic, via the blowing-up of $W$ along $L$, to the strict transform of $W$ minus a parabola $P$. Therefore $\beta(W \backslash L)=\beta\left(\mathbb{A}_{\mathbb{R}}^{1} \times P\right)-\beta(P)=$ $\left(\beta\left(\mathbb{A}_{\mathbb{R}}^{1}\right)-1\right) \beta(P)=(u-1) u$. Finally $\beta(W)=u^{2}$.

Remark 2.11. The virtual Poincaré polynomial satisfies $\operatorname{dim}(A)=\operatorname{deg}(\beta(A))$ for an arc-symmetric set $A$. In particular, it respects the dimension.

Proof of Theorem 2.3. We prove Theorem 2.3 by induction on the dimension; the rank-n inductive hypothesis claims that $\chi$ is defined on arc-symmetric sets of dimension less than or equal to $n$, is invariant under isomorphisms between arc-symmetric sets, and is additive.

For $n=0$ the arc-symmetric sets are just finite unions of points and the result is clearly true. Assume that the inductive hypothesis is true at rank $n-1$. We prove the result at rank $n$ in two steps: 


\section{MotiViC INVARIANTS OF BLOW-NASH EQUIVALENCE}

(1) If $\chi$ is an additive map on the non-singular elements of $\mathcal{A S}$ of dimension less than or equal to $n$, then $\chi$ extends to an additive map on all arc-symmetric sets of dimension less than or equal to $n$.

(2) If $\chi$ satisfies properties (P1), (P2) and (P3), then $\chi$ extends to an additive map on the non-singular elements of $\mathcal{A S}$ of dimension less than or equal to $n$.

Step 1. Let $A \in \mathcal{A S}$ of dimension $n$. There exists a stratification $\bar{A}^{\mathcal{Z}}=\bigcup_{S \in \mathcal{S}} S$ of $\bar{A}^{\mathcal{Z}}$ with non-singular algebraic strata, i.e. $\bar{A}^{\mathcal{Z}}$ is a disjoint union of locally closed algebraic varieties (note in particular that we do not ask the strata to be connected). Then $S \cap A$, for each $S \in \mathcal{S}$, is a non-singular arc-symmetric set, and thus $\chi(S \cap A)$ is defined. Put $\chi(A)=\sum_{S \in \mathcal{S}} \chi(S \cap A)$. One has to check that $\chi(A)$ is well-defined and satisfies the additivity property.

We show first that $\chi(A)$ is equal to $\sum_{S \in \mathcal{S}} \chi(S \cap A)$ in the case where $A$ is non-singular, by induction on the number of elements in $\mathcal{S}$. Indeed, take $N_{0} \in \mathcal{S}$; then

$$
\chi(A)=\chi\left(A \backslash\left(A \cap N_{0}\right)\right)+\chi\left(A \cap N_{0}\right) \quad \text { and } \quad \chi\left(A \backslash\left(A \cap N_{0}\right)\right)=\sum_{S \in \mathcal{S} \backslash\left\{N_{0}\right\}} \chi(S \cap A)
$$

by induction, so the result follows.

Now, if $\mathcal{S}_{1}$ and $\mathcal{S}_{2}$ are two stratifications of $\bar{A}^{\mathcal{Z}}$, one can find a common refinement $\mathcal{S}$ of $\mathcal{S}_{1}$ and $\mathcal{S}_{2}$. The independence in the non-singular case implies that

$$
\sum_{S \in \mathcal{S}_{1}} \chi(S \cap A)=\sum_{S \in \mathcal{S}} \chi(S \cap A)=\sum_{S \in \mathcal{S}_{2}} \chi(S \cap A),
$$

and thus $\chi$ does not depend on the choice of the stratification.

Let us show finally that $\chi$ is additive. Take $A, B \in \mathcal{A S}$, with $B \subset A$, of dimension less than or equal to $n$. One can choose a stratification $\bigcup_{S \in \mathcal{S}} S$ of $\bar{A}^{\mathcal{Z}}$ such that $\bar{B}^{\mathcal{Z}}$ and $\overline{A \backslash B}^{\mathcal{Z}}$ are unions of strata. Then

$$
\sum_{S \in \mathcal{S}} \chi(S \cap B)+\sum_{S \in \mathcal{S}} \chi(S \cap(A \backslash B))=\sum_{S \in \mathcal{S}}(\chi(S \cap B)+\chi(S \cap(A \backslash B))),
$$

and $\chi(S \cap(A \backslash B))+\chi(S \cap B)=\chi(S \cap A)$ because the strata are non-singular, so $\chi$ is additive.

Step 2. The second step constitutes the heart of the work. Define $\chi$ over the non-singular arcsymmetric sets of dimension $n$ in the following way.

(D1) If $A=\bigcup_{i \in I} A_{i}$ denotes the decomposition of $A$ into irreducible components, put $\chi(A)=$ $\sum_{i \in I} \chi\left(A_{i}\right)$.

(D2) If $A \in \mathcal{A S}$ is non-singular and irreducible, then define $\chi(A)$ by $\chi(A)=\chi(\widetilde{A})-\chi(\widetilde{A} \backslash$ $\pi^{-1}(A)$ ), where $\widetilde{A}$ is the connected component of a resolution of singularities $\pi$ of $\bar{A}^{\mathcal{Z}}$ given by Remark 1.15.

We have to prove that $\chi$ is well-defined, invariant under isomorphisms, and additive over the non-singular elements of $\mathcal{A S}$.

The following lemma will be useful in the sequel.

Lemma 2.12. Let $A$ and $B$ in $\mathcal{A S}$ be non-singular, irreducible and isomorphic. Suppose that $\bar{A}^{\mathcal{Z}}$ and $\bar{B}^{\mathcal{Z}}$ are non-singular, and denote by $\widetilde{A} \subset \bar{A}^{\mathcal{Z}}$ and $\widetilde{B} \subset \bar{B}^{\mathcal{Z}}$ the connected components containing $A$ and $B$ respectively. Then

$$
\chi(\widetilde{A})-\chi(\widetilde{A} \backslash A)=\chi(\widetilde{B})-\chi(\widetilde{B} \backslash B) .
$$

Proof. By definition of an isomorphism between arc-symmetric sets, we know that $\bar{A}^{\mathcal{Z}}$ and $\bar{B}^{\mathcal{Z}}$ are birationally equivalent, and the weak factorization theorem [AKMW02, Wlo03] factors this 


\section{G. FICHOU}

birational isomorphism into a succession of blowings-up and blowings-down. In particular, we can assume that the birational isomorphism between $\bar{A}^{\mathcal{Z}}$ and $\bar{B}^{\mathcal{Z}}$ is just a blowing-up $\pi: \bar{A}^{\mathcal{Z}} \longrightarrow \bar{B}^{\mathcal{Z}}$ along a non-singular variety $C$ such that $C \cap B=\emptyset$. Note that $\pi^{-1}(\widetilde{B})=\widetilde{A}$ by Proposition 1.17. Now $\chi(\widetilde{B} \backslash B)=\chi(\widetilde{B} \cap C)+\chi(\widetilde{B} \backslash(B \cup C))$ by the additivity inductive hypothesis because $\operatorname{dim} \widetilde{B} \backslash B$ is strictly less than $\operatorname{dim} B$ by Proposition 1.6. Moreover

$$
\chi(\widetilde{B})-\chi(\widetilde{B} \cap C)=\chi(\widetilde{A})-\chi\left(\widetilde{A} \cap \pi^{-1}(C)\right) \quad \text { and } \quad \chi(\widetilde{B} \backslash(B \cup C))=\chi\left(\widetilde{A} \backslash\left(A \cup \pi^{-1}(C)\right)\right)
$$

by property (P3), and the inductive hypothesis about invariance under isomorphisms respectively. Therefore

$$
\chi(\widetilde{B})-\chi(\widetilde{B} \backslash B)=\chi(\widetilde{A})-\chi\left(\widetilde{A} \cap \pi^{-1}(C)\right)-\chi\left(\widetilde{A} \backslash\left(A \cup \pi^{-1}(C)\right)\right),
$$

which is equal to $\chi(\widetilde{A})-\chi(\widetilde{A} \backslash A)$ by the additivity inductive hypothesis.

Now we return to step 2 of the proof of Theorem 2.3. Let us check that the definition of $\chi$, for the non-singular and irreducible arc-symmetric sets of dimension $n$, does not depend on the choice of the resolution of singularities of Remark 1.15.

Let $A \in \mathcal{A S}$ be non-singular and irreducible, and let $\pi_{i}: \widetilde{X}_{i} \longrightarrow \bar{A}^{\mathcal{Z}}$, for $i \in\{1,2\}$, be resolutions of singularities of $\bar{A}^{\mathcal{Z}}$. Let $\widetilde{A_{i}}$ be the connected components of $\widetilde{X}_{i}$ given by Proposition 1.13. One has to show that: $\chi\left(\widetilde{A_{1}}\right)-\chi\left(\widetilde{A_{1}} \backslash \pi_{1}^{-1}(A)\right)=\chi\left(\widetilde{A_{2}}\right)-\chi\left(\widetilde{A_{2}} \backslash \pi_{2}^{-1}(A)\right)$.

But $\pi_{1}^{-1}(A)$ and $\pi_{2}^{-1}(A)$ are isomorphic irreducible non-singular arc-symmetric sets because $\pi_{i}$ is an isomorphism on a Zariski open subset of $\widetilde{X}_{i}$ containing $\pi_{i}^{-1}(A)$, for $i \in\{1,2\}$. Therefore Lemma 2.12 applies and $\chi$ is well-defined.

Now let us show that $\chi$ is invariant under isomorphisms between arc-symmetric sets. The proof is very similar to the last one. Let $A$ and $B$ in $\mathcal{A S}$ be non-singular, irreducible and isomorphic. Then there exist Zariski open subsets $U$ and $V$ in $\bar{A}^{\mathcal{Z}}$ and $\bar{B}^{\mathcal{Z}}$ respectively, and an algebraic isomorphism $\phi: U \longrightarrow V$ such that $\phi(A)=B$. Choose resolutions of singularities

$$
\pi_{A}: \widetilde{X} \longrightarrow \bar{A}^{\mathcal{Z}} \text { and } \pi_{B}: \tilde{Y} \longrightarrow \bar{B}^{\mathcal{Z}}
$$

for $\bar{A}^{\mathcal{Z}}$ and $\bar{B}^{\mathcal{Z}}$ respectively. Then $\pi_{A}^{-1}(A)$ and $\pi_{B}^{-1}(B)$ are isomorphic as arc-symmetric sets, and then by Lemma 2.12: $\chi\left(\pi_{A}^{-1}(A)\right)=\chi\left(\pi_{B}^{-1}(B)\right)$.

Moreover $\chi\left(\pi_{A}^{-1}(A)\right)$ equals $\chi(A)$ because both are equal to $\chi(\widetilde{A})-\chi\left(\widetilde{A} \backslash \pi_{A}^{-1}(A)\right)$, where $\widetilde{A}$ is the connected component of $\widetilde{X}$ given by Proposition 1.13. In the same way $\chi\left(\pi_{B}^{-1}(B)\right)$ is equal to $\chi(B)$, hence the equalities $\chi(A)=\chi\left(\pi_{A}^{-1}(A)\right)=\chi\left(\pi_{B}^{-1}(B)\right)=\chi(B)$ hold.

In the case where $A$ and $B$ are not irreducible, it suffices to decompose $A$ and $B$ into irreducible components, and to apply the property (D1) because an isomorphism between arc-symmetric sets respects the irreducible components.

Finally, let us check that $\chi$ is additive. Let $B \subset A$ be an inclusion of non-singular arc-symmetric sets. Note that, by definition of $\chi$, we need to prove the result only in the case where $A$ is irreducible.

If $A$ and $B$ have the same dimension, then $\bar{B}^{\mathcal{A S}}=\bar{A}^{\mathcal{A S}}$ by Proposition 1.7 , and so $\bar{B}^{\mathcal{Z}}=\bar{A}^{\mathcal{Z}}$. Now choose a resolution of singularities $\pi: \widetilde{X} \longrightarrow \bar{A}^{\mathcal{Z}}$ for $\bar{A}^{\mathcal{Z}}$. If $\tilde{A}$ denotes the connected component of $\widetilde{X}$ given by Proposition 1.13 for $A$, then it is also the component associated to $B$, and therefore $\chi(B)=\chi(\widetilde{A})-\chi\left(\widetilde{A} \backslash \pi^{-1}(B)\right)$.

Now $\chi\left(\widetilde{A} \backslash \pi^{-1}(B)\right)=\chi\left(\widetilde{A} \backslash \pi^{-1}(A)\right)+\chi\left(\pi^{-1}(A) \backslash \pi^{-1}(B)\right)$ by the inductive hypothesis on additivity. As a consequence $\chi(B)$ equals $\chi(A)-\chi(A \backslash B)$ because $\chi\left(\pi^{-1}(A) \backslash \pi^{-1}(B)\right)$ is equal to $\chi(A \backslash B)$ by the invariance under isomorphisms in dimension smaller that $n$.

If $\operatorname{dim} B<\operatorname{dim} A$, choose a resolution of singularities $\pi: \widetilde{X} \longrightarrow \bar{A}^{\mathcal{Z}}$ for $\bar{A}^{\mathcal{Z}}$. It is also a resolution of singularities of $\overline{A \backslash B}^{\mathcal{Z}}=\bar{A}^{\mathcal{Z}}$ and so $\chi(A \backslash B)$ equals the difference $\chi(\widetilde{A})-\chi\left(\widetilde{A} \backslash \pi^{-1}(A \backslash B)\right)$. 


\section{MotiviC INVARIANTS OF BLOW-NASH EQUIVALENCE}

Now

$$
\chi\left(\widetilde{A} \backslash \pi^{-1}(A \backslash B)\right)=\chi\left(\left(\widetilde{A} \backslash \pi^{-1}(A)\right) \cup \pi^{-1}(B)\right)=\chi\left(\widetilde{A} \backslash \pi^{-1}(A)\right)+\chi\left(\pi^{-1}(B)\right)
$$

by the inductive assumption, and once more by the inductive assumption $\chi\left(\pi^{-1}(B)\right)$ is equal to $\chi(B)$. Finally $\chi(A \backslash B)=\chi(A)-\chi(B)$, which completes the proof of step 2, and thus the proof of Theorem 2.3.

As was the case for the previous proof, we are going to prove Theorem 2.7 by induction on the dimension. The following relations will be useful:

(1) $\chi\left(\bigsqcup_{i=1}^{k} A_{i}\right)=\sum_{i=1}^{k} \chi\left(A_{i}\right)$, where the union of the arc-symmetric sets $A_{i}, i=1, \ldots, k$, is disjoint; and

(2) $\chi(A)=\underset{\widetilde{A}}{\chi}(\widetilde{A})-\chi(\widetilde{A} \backslash A)$, where $A$ is a non-singular arc-symmetric set whose arc-symmetric closure $\widetilde{A}$ is non-singular.

Proof of Theorem 2.7. Assume, as an inductive hypothesis at rank $n$, that $\chi$ is multiplicative for all arc-symmetric sets of dimension strictly less than or equal to $n$.

We remark that we can restrict our attention to the non-singular case because, by considering stratifications of arc-symmetric sets with non-singular strata, we prove the multiplicativity directly with formula (1) above.

Assume therefore that $A$ and $B$ are non-singular arc-symmetric sets of dimension less than or equal to $n$; suppose that $\operatorname{dim} A=n$ for instance.

In the case where $A$ is compact, the result follows from another induction, which is finite, on the dimension of $B$ : indeed, resolving the singularities of $\bar{B}^{\mathcal{Z}}$, one can assume that $B \subset \widetilde{B}$, where $\widetilde{B}$ is the non-singular arc-symmetric closure of $B$. Then, by formula $(2), \chi(A \times B)$ is equal to $\chi(A \times \widetilde{B})-\chi(A \times(\widetilde{B} \backslash B))$. However, $\chi(A \times \widetilde{B})=\chi(A) \chi(\widetilde{B})$, for they are compact and nonsingular, and $\chi(A \times(\widetilde{B} \backslash B))=\chi(A) \chi(\widetilde{B} \backslash B)$, as we can see by stratifying $\widetilde{B} \backslash B$ with non-singular strata and using the inductive assumption of the second induction, because $\operatorname{dim} \widetilde{B} \backslash B<\operatorname{dim} B$ by Proposition 1.6. Consequently

$$
\chi(A \times B)=\chi(A)(\chi(\widetilde{B})-\chi(\widetilde{B} \backslash B))=\chi(A) \chi(B) .
$$

If $A$ is no longer compact, then compactify $A$ and $B$ in $\widetilde{A}$ and $\widetilde{B}$ respectively, and assume that $\widetilde{A}, \widetilde{B}$ are non-singular, even if it means resolving singularities, as before.

Then, by additivity, $\chi(A \times B)=\chi(\widetilde{A} \times B)-\chi((\widetilde{A} \backslash A) \times \widetilde{B})+\chi((\widetilde{A} \backslash A) \times(\widetilde{B} \backslash B))$.

The multiplicativity of the first two terms comes from the preceding case (for the second term, stratify the possibly singular set $\widetilde{A} \backslash A$ ), and the multiplicativity of the third is obtained by the inductive assumption for $\max (\operatorname{dim} \widetilde{A} \backslash A, \operatorname{dim} \widetilde{B} \backslash B)<n$ by Proposition 1.6. Therefore $\chi(A \times B)=$ $\chi(\widetilde{A}) \chi(B)-\chi(\widetilde{A} \backslash A) \chi(\widetilde{B})+\chi(\widetilde{A} \backslash A) \chi(\widetilde{B} \backslash B)=\chi(A) \chi(B)$, and Theorem 2.7 is proved.

\subsection{Virtual Betti numbers and Nash isomorphisms}

The definition of an isomorphism between arc-symmetric sets, which we gave in $\S 1$, is algebraic, via birational morphisms. But arc-symmetric sets are also closely related to analytic objects. As an example, the following proposition emphasizes the good behaviour of the virtual Poincaré polynomial $\beta$ with respect to the compact algebraic varieties that are non-singular as analytic varieties. Recall that by $b_{i}(X)$ we denote the $i$ th Betti number of $X$ with coefficients in $\mathbb{Z}_{2}$, and let us put $b(X)=\sum_{i=0}^{\operatorname{dim} X} b_{i}(X) u^{i}$.

Proposition 2.13. Let $X$ be a compact algebraic variety that is non-singular as an analytic space. Then the virtual Poincaré polynomial $\beta$ evaluated at $X$ is equal to $b(X)$. 


\section{G. FICHOU}

Proof. One can desingularize the algebraic singularities of $X$ by a sequence of blowings-up with smooth centres [BM97, Hir64]. At each step of the desingularization, one has the following relations, where $B l_{C} X$ designates the blowing-up of $X$ along the non-singular subvariety $C$, and $E$ is the exceptional divisor: $\beta\left(B l_{C} X\right)-\beta(E)=\beta(X)-\beta(C)$, because the blowing-up is birational, and $b\left(B l_{C} X\right)-b(E)=b(X)-b(C)$, because $X$ and $C$ are smooth and the blowing-up is a degree-one morphism (cf. Corollary 2.5).

We remark that $\beta(E)$ and $\beta(C)$ are equal to $b(E)$ and $b(C)$ respectively by definition of $\beta$, because $E$ and $C$ are non-singular and compact arc-symmetric sets. The same is true for $\widetilde{X}$, the desingularization of $X$. Then $\beta(X)$ and $b(X)$ can be expressed by the same formulae in terms of $\beta$ for the former, and $b$ for the latter, where the spaces involved are non-singular and compact. Therefore, for each of these spaces, $\beta$ and $b$ coincide, and then $\beta(X)$ is equal to $b(X)$.

Remark 2.14.

(i) Note that a real algebraic variety that is non-singular as an analytic space is not necessarily non-singular as an algebraic variety (cf. [BCR98, Example 3.3.12.b]).

(ii) We will see in the proof of Theorem 2.16 that the assumption ' $X$ is an algebraic variety' can be replaced by the weaker ' $X$ is a semi-algebraic set'.

In order to relate the analytic aspect of arc-symmetric sets to the behaviour of the virtual Poincaré polynomial $\beta$, we propose the following definition of a Nash isomorphism between arc-symmetric sets.

Definition 2.15. Let $A, B \in \mathcal{A S}$. Assume that there exist compact analytic varieties $V_{1}, V_{2}$ containing $A, B$ respectively, and also an analytic isomorphism $\phi$ from $V_{1}$ to $V_{2}$ such that $\phi(A)=B$. If moreover one can choose $V_{1}, V_{2}$ to be semi-algebraic sets and $\phi$ to be a semi-algebraic map, then we say that $A$ and $B$ are Nash isomorphic.

Theorem 2.16. Nash isomorphic arc-symmetric sets have the same value under the virtual Poincaré polynomial.

Proof. Once more, we are going to prove the result by induction on the dimension. As a first step, let us generalize the result of Proposition 2.13.

Step 1. Let $A$ be a compact arc-symmetric set that is also a non-singular analytic subspace of the Zariski closure $X$ of $A$. Then $\beta(A)$ is equal to $b(A)$.

In order to prove this claim, one would want to apply the same method as in the proof of Proposition 2.13. But, if $C$ is a smooth centre of blowing-up for $X$, it is not true in general that $C \cap A$ is still non-singular, so the equality $\beta(C \cap A)=b(C \cap A)$ no longer holds. In order to solve this problem, consider the algebraic normalization $\widetilde{X}$ of $X$. There exists $\widetilde{A} \subset \widetilde{X}$ the analytic normalization of $A$ (see [Loj91]), which is analytically isomorphic to $A$ because $A$ is non-singular as an analytic space. Then $b(A)$ is equal to $b(\widetilde{A})$ because $b$ is invariant under homeomorphisms.

Moreover $\beta(A)$ is equal to $\beta(\widetilde{A})$; in fact, the algebraic normalization is a birational map; hence it is an algebraic isomorphism outside compact subvarieties $E$ and $D$ of $\widetilde{X}$ and $X$ respectively, of dimension strictly less than $\operatorname{dim} X=\operatorname{dim} A$. Thus $\beta(\widetilde{A} \backslash E)$ is equal to $\beta(A \backslash D)$ by Corollary 2.5 , and the algebraic normalization, restricted to $\widetilde{A} \cap E$, is an analytic isomorphism onto $A \cap D$, so $\beta(\widetilde{A} \cap E)$ is equal to $\beta(A \cap D)$ by the inductive assumption.

Note that $\widetilde{X}$ is locally analytically irreducible as a normal space, and therefore $\widetilde{A}$ is a union of connected components of $\widetilde{X}$. Now it is true that $C \cap \widetilde{A}$ is non-singular when $C$ is non-singular, and the method of the proof of Proposition 2.13 applies; therefore $\beta(\widetilde{A})$ is equal to $b(\widetilde{A})$. It follows that $\beta(A)=\beta(\widetilde{A})=b(\widetilde{A})=b(A)$, and then step 1 is completed. 


\section{MotiviC INVARIANTS OF BLOW-NASH EQUIVALENCE}

Step 2. Let $A_{1}$ and $A_{2}$ be Nash isomorphic arc-symmetric sets. Let us prove the theorem in the particular case where $A_{1}$ and $A_{2}$ are non-singular arc-symmetric sets and moreover, with the assumptions of the definition of a Nash isomorphism, the compact analytic varieties $V_{1}$ and $V_{2}$ are supposed to be smooth as analytic spaces.

(i) First we show that $\beta\left({\overline{A_{2}}}^{\mathcal{A S}}\right)=\beta\left({\overline{A_{1}}}^{\mathcal{A S}}\right)$. We remark that ${\overline{A_{2}}}^{\mathcal{A S}}$ is a union of connected components of $V_{2}$ by Proposition 1.7. Thus $\bar{A}_{2}^{\mathcal{A S}}$ is also non-singular as an analytic variety and $\beta\left({\overline{A_{2}}}^{\mathcal{A S}}\right)$ is equal to $b\left({\overline{A_{2}}}^{\mathcal{A S}}\right)$ by step 1 .

Moreover ${\overline{A_{2}}}^{\mathcal{A S}}$ is isomorphic to ${\overline{A_{1}}}^{\mathcal{A S}}$ by $\phi$. Indeed, $\phi^{-1}\left({\overline{A_{2}}}^{\mathcal{A S}}\right)$ is a closed arc-symmetric set because $\phi$ has an arc-symmetric graph and $\phi$ is continuous, and it contains $A_{1}$, so $\overline{A_{1}} \mathcal{A S} \subset$ $\phi^{-1}\left({\overline{A_{2}}}^{\mathcal{A S}}\right)$. The reverse inclusion comes from the fact that the image of an arc-symmetric set by an injective map with arc-symmetric graph is still an arc-symmetric set (recall that $\mathcal{A S}$ forms a constructible category, cf. $\S 1$ ). Consequently, ${\overline{A_{1}}}^{\mathcal{A S}}$ is non-singular as an analytic variety because so is ${\overline{A_{2}}}^{\mathcal{A S}}$ and $\phi$ is an analytic isomorphism; hence $\beta\left({\overline{A_{1}}}^{\mathcal{A S}}\right)$ equals $b\left({\overline{A_{1}}}^{\mathcal{A S}}\right)$ by step 1.

We remark also that $b\left({\overline{A_{2}}}^{\mathcal{A S}}\right)$ is equal to $b\left({\overline{A_{1}}}^{\mathcal{A S}}\right)$ because $\phi$ is a homeomorphism between these two smooth compact topological varieties.

These equalities imply that $\beta\left({\overline{A_{2}}}^{\mathcal{A S}}\right)$ equals $\beta\left({\overline{A_{1}}}^{\mathcal{A S}}\right)$.

(ii) Then, we remark that $\beta\left({\overline{A_{1}}}^{\mathcal{A S}} \backslash A_{1}\right)$ is equal to $\beta\left({\overline{A_{2}}}^{\mathcal{A S}} \backslash A_{2}\right)$. Indeed this follows from the inductive hypothesis, for ${\overline{A_{1}}}^{\mathcal{A S}} \backslash A_{1}$ and ${\overline{A_{2}}}^{\mathcal{A S}} \backslash A_{2}$ are Nash isomorphic arc-symmetric sets of dimension strictly less than $\operatorname{dim} A_{2}$.

(iii) Finally $\beta\left(A_{1}\right)$ is equal to $\beta\left(A_{2}\right)$. Actually $\beta\left(A_{1}\right)=\beta\left({\overline{A_{1}}}^{\mathcal{A S}}\right)-\beta\left({\overline{A_{1}}}^{\mathcal{A S}} \backslash A_{1}\right)$ and $\beta\left(A_{2}\right)=$ $\beta\left({\overline{A_{2}}}^{\mathcal{A S}}\right)-\beta\left({\overline{A_{2}}}^{\mathcal{A S}} \backslash A_{2}\right)$, and we have proved that the second members are equal, so $\beta\left(A_{1}\right)$ equals $\beta\left(A_{2}\right)$ by additivity of the virtual Poincaré polynomial $\beta$.

Step 3. Reduction of the problem to step 2. Let $A_{1}$ and $A_{2}$ be Nash isomorphic arc-symmetric sets. By definition of a Nash isomorphism, there exist compact analytic varieties $V_{1}$ and $V_{2}$ containing $A$ and $B$ respectively, and an analytic isomorphism $\phi: V_{1} \longrightarrow V_{2}$ such that $\phi\left(A_{1}\right)=A_{2}$, and moreover $V_{1}$ and $V_{2}$ are semi-algebraic sets and $\phi$ is a semi-algebraic map.

Denote by $X_{1}$ and $X_{2}$ the Zariski closures of $V_{1}$ and $V_{2}$ respectively.

As a first step, we are going to obtain a regular morphism rather than a semi-algebraic map between $V_{1}$ and $V_{2}$. Denote by $\Gamma$ the graph of $\phi$. This graph is semi-algebraic and analytic, thus arcsymmetric. Then the projection $p_{i}$ from $Z=\bar{\Gamma}^{\mathcal{Z}}$ onto $X_{i}$, for $i \in\{1,2\}$, is a regular morphism whose restriction to $\Gamma$ is an analytic isomorphism onto $V_{i}$. Moreover, the preimages by these restrictions of $A_{1}$ and $A_{2}$ coincide, so one can put $B=p^{-1}(A)$, where $A=A_{i} \subset V_{i}=V \subset X_{i}=X$ for $i \in\{1,2\}$ and $p: Z \longrightarrow X$ denotes the natural projection. Therefore $B$ is an arc-symmetric set that is Nash isomorphic to $A$, and the issue is now to prove that $\beta(B)$ equals $\beta(A)$.

In order to do this, we want to come down to non-singular objects.

Desingularize $X$ by a sequence of blowings-up with respect to coherent algebraic sheaves of ideals (this is possible by [BM97, Hir64]). By blowing-up $Z$ with respect to the corresponding inverse image ideal sheaves with respect to $p$, at each step one has a regular morphism which lifts the projection $p: Z \longrightarrow X$ to the corresponding blowing-up by the universal property of algebraic blowing-up. Let $\pi_{X}: \widetilde{X} \longrightarrow X$ denote a resolution of singularities of $X$ and $\pi_{Z}: \widetilde{Z} \longrightarrow Z$ the corresponding composition of blowings-up of $Z$. If $\widetilde{p}$ denotes the morphism obtained between $\widetilde{Z}$ and $\widetilde{X}$ by the 


\section{G. FICHOU}

universal property, one has the diagram

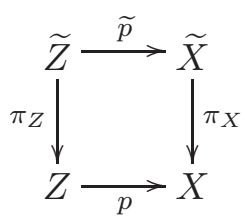

which is commutative. Moreover $\widetilde{p}$ restricted to the analytic strict transform $\widetilde{\Gamma}$ of $\Gamma$ is an analytic isomorphism onto the strict transform $\widetilde{V}$ of $V$ because so is $p$ between $\Gamma$ and $V$ (here we consider the blowing-up as an analytic one).

Now we reduce the problem to the case where $A$ and $B$ are non-singular by the inductive hypothesis. In fact, the singular parts of $A$ and $B$ are not necessarily exchanged by $p_{\mid \Gamma}$, but

$$
\operatorname{Sing}(A) \cup p_{\mid \Gamma}^{-1}(\operatorname{Sing}(B)) \quad \text { and } \quad \operatorname{Sing}(B) \cup p_{\mid \Gamma}(\operatorname{Sing}(A))
$$

are exchanged by $p_{\mid \Gamma}$ and its inverse, and thus are Nash isomorphic. Moreover the dimension of these arc-symmetric sets is strictly less than $\operatorname{dim} A=\operatorname{dim} B$, so they have the same image by $\beta$ due to the inductive hypothesis. Let us denote by $A^{\prime}$ and $B^{\prime}$ the respective complements of these sets in $A$ and $B$. Now $A^{\prime}$ and $B^{\prime}$ are non-singular.

As $A^{\prime}$ is non-singular, it is isomorphic to its preimage in the desingularization $\widetilde{X}$ of $X$, in the sense of Definition 1.10. Consequently $B^{\prime}$ is also isomorphic to its preimage in $\widetilde{\Gamma}$ by commutativity of the diagram. As a consequence $\beta\left(A^{\prime}\right)$ and $\beta\left(B^{\prime}\right)$ are equal to $\beta\left(\pi^{-1}\left(A^{\prime}\right)\right)$ and $\beta\left(\pi^{-1}\left(B^{\prime}\right)\right)$ respectively, and we have reduced the problem to step 2. The proof of Theorem 2.16 is complete.

\section{Zeta functions}

The zeta functions of a real analytic function germ, which we consider in this section, are directly inspired by the work of Denef and Loeser [DL01] on their motivic zeta functions. In particular, our zeta functions are defined by considering the image, under the virtual Poincaré polynomial, of certain constructible real algebraic subsets of the arc space of an affine space.

In the case where the function germ is Nash (that is, semi-algebraic and analytic), we connect these zeta functions with the blow-analytic equivalence in $\S 4$.

In this section, after the definition and some examples of zeta functions, we focus on the Denef and Loeser formulae. These formulae enable one to compute the zeta functions in terms of a modification of the Nash function germ we consider. It gives some possibilities to compute these zeta functions more easily, and it is also a key ingredient for the application to blow-Nash equivalence in $\S 4$.

Note that we state also a Thom-Sebastiani formula for analytic germs of the same sign, but we do not obtain such a formula in the general case.

\subsection{Zeta functions and the Denef and Loeser formula}

We first define the zeta functions for a germ of real analytic functions. Then we give a formula to compute these zeta functions in terms of a modification in the case when the germ is Nash.

Denote by $\mathcal{L}$ the space of arcs at the origin $0 \in \mathbb{R}^{d}$, defined by

$$
\mathcal{L}=\mathcal{L}\left(\mathbb{R}^{d}, 0\right)=\left\{\gamma:(\mathbb{R}, 0) \longrightarrow\left(\mathbb{R}^{d}, 0\right): \gamma \text { formal }\right\},
$$

and by $\mathcal{L}_{n}$ the space of arcs truncated at the order $n+1$,

$$
\mathcal{L}_{n}=\mathcal{L}_{n}\left(\mathbb{R}^{d}, 0\right)=\left\{\gamma \in \mathcal{L}: \gamma(t)=a_{1} t+a_{2} t^{2}+\cdots+a_{n} t^{n}, a_{i} \in \mathbb{R}^{d}\right\},
$$




\section{MotiviC INVARIANTS OF BLOW-NASH EQUIVALENCE}

for $n \geqslant 0$ an integer. Let $\pi_{n}: \mathcal{L} \longrightarrow \mathcal{L}_{n}$ and $\pi_{n, i}: \mathcal{L}_{n} \longrightarrow \mathcal{L}_{i}$, with $n \geqslant i$, be the truncation morphisms.

Consider $f:\left(\mathbb{R}^{d}, 0\right) \longrightarrow(\mathbb{R}, 0)$ a real analytic function germ. We define the naive zeta function $Z_{f}(T)$ of $f$ as the following element of $\mathbb{Z}\left[u, u^{-1}\right][[T]]$ :

$$
Z_{f}(T)=\sum_{n \geqslant 1} \beta\left(\mathcal{X}_{n}\right) u^{-n d} T^{n}
$$

where

$$
\mathcal{X}_{n}=\left\{\gamma \in \mathcal{L}_{n}: \operatorname{ord}(f \circ \gamma)=n\right\}=\left\{\gamma \in \mathcal{L}_{n}: f \circ \gamma(t)=b t^{n}+\cdots, b \neq 0\right\} .
$$

Similarly, we define zeta functions with sign, by

$$
Z_{f}^{+}(T)=\sum_{n \geqslant 1} \beta\left(\mathcal{X}_{n}^{+}\right) u^{-n d} T^{n} \quad \text { and } \quad Z_{f}^{-}(T)=\sum_{n \geqslant 1} \beta\left(\mathcal{X}_{n}^{-}\right) u^{-n d} T^{n}
$$

where

$$
\mathcal{X}_{n}^{+}=\left\{\gamma \in \mathcal{L}_{n}: f \circ \gamma(t)=+t^{n}+\cdots\right\} \quad \text { and } \quad \mathcal{X}_{n}^{-}=\left\{\gamma \in \mathcal{L}_{n}: f \circ \gamma(t)=-t^{n}+\cdots\right\} .
$$

We remark that $\mathcal{X}_{n}$ and $\mathcal{X}_{n}^{ \pm}$, for $n \geqslant 1$, are Zariski constructible subsets of $\mathbb{R}^{n d}$, and hence belong to $\mathcal{A S}$.

Example 3.1. Let $f:(\mathbb{R}, 0) \longrightarrow(\mathbb{R}, 0)$ be defined by $f(x)=x^{k}, k \geqslant 1$. Then

$$
\mathcal{X}_{n}= \begin{cases}\left\{\gamma=a_{m} t^{m}+\cdots+a_{n} t^{n} ; a_{m} \neq 0\right\} \simeq \mathbb{R}^{*} \times \mathbb{R}^{n-m} & \text { if } n=m k, \\ \emptyset & \text { otherwise. }\end{cases}
$$

Therefore $\beta\left(\mathcal{X}_{n}\right)=(u-1) u^{n-m}$ if $n=m k$ and 0 otherwise hence

$$
Z_{f}(T)=\sum_{m \geqslant 1}(u-1) u^{m k-m}\left(\frac{T}{u}\right)^{m k}=(u-1) \frac{T^{k}}{u-T^{k}} .
$$

To compute the zeta functions with sign, we have to consider the cases $k=2 p$ and $k=2 p+1$.

If $k=2 p$, then $\mathcal{X}_{n}^{-}=\emptyset$ and

$$
\mathcal{X}_{n}^{+}= \begin{cases}\left\{\gamma= \pm t^{m}+\cdots+a_{n} t^{n} ; a_{m} \neq 0\right\} \simeq\{ \pm 1\} \times \mathbb{R}^{n-m} & \text { if } n=m k \\ \emptyset & \text { otherwise }\end{cases}
$$

so

$$
Z_{f}^{+}(T)=\sum_{m \geqslant 1} 2 u^{m k-m}\left(\frac{T}{u}\right)^{m k}=2 \frac{T^{k}}{u-T^{k}}
$$

If $k=2 p+1$, then

$$
\mathcal{X}_{n}^{ \pm}= \begin{cases}\left\{\gamma= \pm t^{m}+\cdots+a_{n} t^{n} ; a_{m} \neq 0\right\} \simeq\{ \pm 1\} \times \mathbb{R}^{n-m} & \text { if } n=m k \\ \emptyset & \text { otherwise }\end{cases}
$$

and thus

$$
Z_{f}^{+}(T)=Z_{f}^{-}(T)=\sum_{m \geqslant 1} u^{m k-m}\left(\frac{T}{u}\right)^{m k}=\frac{T^{k}}{u-T^{k}} .
$$

It may be convenient to express the zeta functions of a germ $f$ in terms of a modification of $f$, that is a proper birational map, which is an isomorphism over the complement of the zero locus of $f$, and such that $f$, being composed with the modification, becomes a function with only normal crossings. Similarly to [DL99], the following proposition, called the Denef and Loeser formula, enables one to do this in the case where the germ is Nash. 


\section{G. FICHOU}

Proposition 3.2 (Denef and Loeser formula). Let $f:\left(\mathbb{R}^{d}, 0\right) \longrightarrow(\mathbb{R}, 0)$ be a Nash function germ. Let $\sigma:\left(M, \sigma^{-1}(0)\right) \longrightarrow\left(\mathbb{R}^{d}, 0\right)$ be a modification of $\mathbb{R}^{d}$ such that $f \circ \sigma$ and the jacobian determinant jac $\sigma$ have only normal crossings simultaneously, and assume moreover that $\sigma$ is an isomorphism over the complement of the zero locus of $f$.

Let $(f \circ \sigma)^{-1}(0)=\bigcup_{j \in J} E_{j}$ be the decomposition into irreducible components of $(f \circ \sigma)^{-1}(0)$, and assume that $\sigma^{-1}(0)=\bigcup_{k \in K} E_{k}$ for some $K \subset J$.

Put $N_{i}=\operatorname{mult}_{E_{i}} f \circ \sigma$ and $\nu_{i}=1+\operatorname{mult}_{E_{i}}$ jac $\sigma$, and for $I \subset J$ denote by $E_{I}^{0}$ the set $\left(\bigcap_{i \in I} E_{i}\right) \backslash\left(\bigcup_{j \in J \backslash I} E_{j}\right)$. Then

$$
Z_{f}(T)=\sum_{I \neq \emptyset}(u-1)^{|I|} \beta\left(E_{I}^{0} \cap \sigma^{-1}(0)\right) \prod_{i \in I} \frac{u^{-\nu_{i}} T^{N_{i}}}{1-u^{-\nu_{i}} T^{N_{i}}} .
$$

Remark 3.3. We have to assume that $f$ is Nash, and not only analytic, in order for the sets $E_{I}^{0}$ to be arc-symmetric.

Example 3.4. Let $f_{k}:\left(\mathbb{R}^{2}, 0\right) \longrightarrow(\mathbb{R}, 0)$ be defined by $f_{k}(x, y)=x^{k}+y^{k}, k \geqslant 2$. The blowing-up at the origin gives a suitable modification $\sigma$ for $f$. Here $(f \circ \sigma)^{-1}(0)$ consists of just the exceptional divisor $\mathbb{P}^{1}$ in the case $k$ even, and furthermore, in the case $k$ odd, of the strict transform of $f$ which is a smooth curve crossing transversally the exceptional divisor. Then

$$
Z_{f_{k}}= \begin{cases}\left(u^{2}-1\right) u^{-2} \frac{T^{k}}{1-u^{-2} T^{k}} & \text { if } k \text { is even, } \\ (u-1) \frac{u^{-2} T^{k}}{1-u^{-2} T^{k}}\left(u+(u-1) \frac{u^{-1} T}{1-u^{-1} T}\right) & \text { if } k \text { is odd. }\end{cases}
$$

Note in particular that, for $k \neq k^{\prime}$, the zeta functions $Z_{f_{k}}$ and $Z_{f_{k^{\prime}}}$ are different.

When we are dealing with signs, one has to define coverings $\widetilde{E_{I}^{0, \pm}}$ of $E_{I}^{0}$, where \pm denotes either + or - , in order to state the corresponding Denef and Loeser formula.

Let $U$ be an affine open subset of $M$ such that $f \circ \sigma=u \prod_{i \in I} y_{i}^{N_{i}}$ on $U$, where $u$ is a unit. Let us put

$$
R_{U}^{ \pm}=\left\{(x, t) \in\left(E_{I}^{0} \cap U\right) \times \mathbb{R} ; t^{m}= \pm \frac{1}{u(x)}\right\}
$$

where $m=\operatorname{gcd}\left(N_{i}\right)$. Then the $R_{U}^{ \pm}$glue together along the $E_{I}^{0} \cap U$ (cf. Lemma 3.12) to give $\widetilde{E_{I}^{0, \pm}}$.

Proposition 3.5. With the assumptions and notations of Proposition 3.2, one can express the zeta functions with sign in terms of a modification as

$$
Z_{f}^{ \pm}(T)=\sum_{I \neq \emptyset}(u-1)^{|I|-1} \beta\left(\widetilde{E_{I}^{0, \pm}} \cap \sigma^{-1}(0)\right) \prod_{i \in I} \frac{u^{-\nu_{i}} T^{N_{i}}}{1-u^{-\nu_{i}} T^{N_{i}}} .
$$

Propositions 3.2 and 3.5 will be proven in $\S 3.2$.

Example 3.6.

(i) The case of a normal crossings function is particularly simple to handle. Let $f:\left(\mathbb{R}^{d}, 0\right) \longrightarrow$ $(\mathbb{R}, 0)$ be defined by $f(x)=u(x) \prod_{i=1}^{k} x_{i}^{N_{i}}$, with $N_{i} \in \mathbb{N}$. Then

$$
Z_{f}(T)=(u-1)^{k} \prod_{i=1}^{k} \frac{u^{-1} T^{N_{i}}}{1-u^{-1} T^{N_{i}}} .
$$




\section{MotiviC INVARIANTS OF BLOW-NASH EQUIVALENCE}

Now, if there exists at least one $N_{i}$ odd, then

$$
Z_{f}^{+}(T)=Z_{f}^{-}(T)=\frac{1}{u-1} Z_{f}(T) .
$$

On the other hand, if all the $N_{i}$ are even, then $Z_{f}^{-}(T)=0$ and

$$
Z_{f}^{+}(T)=\frac{2}{u-1} Z_{f}(T)
$$

if $u$ is positive, the converse otherwise.

(ii) Let $f:\left(\mathbb{R}^{2}, 0\right) \longrightarrow(\mathbb{R}, 0)$ be defined by $f(x, y)=x^{2}+y^{2}$. As $f$ is a positive function, then $Z_{f}^{-}(T)=0$. We obtain a modification in the same way as in Example 3.4, and $\widetilde{E_{I}^{0,+}}$ here is the boundary of a Mobius band, hence homeomorphic to $\mathbb{P}^{1}$. Therefore

$$
Z_{f}^{+}(T)=(u+1) \frac{u^{-2} T^{2}}{1-u^{-2} T^{2}}=\frac{1}{u-1} Z_{f}(T) .
$$

(iii) Let $f:\left(\mathbb{R}^{2}, 0\right) \longrightarrow(\mathbb{R}, 0)$ be defined by $f(x, y)=x^{2}+y^{4}$. One can resolve the singularities of $f$ by two successive blowings-up, and then one obtains that the exceptional divisor $E$ has two irreducible components $E_{1}$ and $E_{2}$ with $N_{1}=2, \nu_{1}=2, N_{2}=4, \nu_{2}=3$. Therefore

$$
Z_{f}(T)=(u-1)^{2} \frac{u^{-2} T^{2}}{1-u^{-2} T^{2}} \frac{u^{-3} T^{4}}{1-u^{-3} T^{4}}+(u-1) u \frac{u^{-2} T^{2}}{1-u^{-2} T^{2}}+(u-1) u \frac{u^{-3} T^{4}}{1-u^{-3} T^{4}} .
$$

Moreover in this case $\widetilde{E_{\{1\}}^{0,+}}$ and $\widetilde{E_{\{2\}}^{0,+}}$ are homeomorphic to a circle minus two points, so

$$
Z_{f}^{+}(T)=2(u-1) \frac{u^{-2} T^{2}}{1-u^{-2} T^{2}} \frac{u^{-3} T^{4}}{1-u^{-3} T^{4}}+(u-1) \frac{u^{-2} T^{2}}{1-u^{-2} T^{2}}+(u-1) \frac{u^{-3} T^{4}}{1-u^{-3} T^{4}} .
$$

Note that in this particular case one has neither $Z_{f}(T)=(u-1) Z_{f}^{+}(T)$ nor $Z_{f}(T)=$ $\frac{1}{2}(u-1) Z_{f}^{+}(T)$, whereas this was the case in the previous examples.

Remark 3.7. It would be convenient to dispose of a Thom-Sebastiani formula in order to compute the zeta functions of the function $f * g$, which is defined by the formula $f * g(x, y)=f(x)+g(y)$, from those of $f$ and $g$, as is the case in [KP03, DL01, Loo02]. Unfortunately, I do not see how to do this in general. However, in the particular case of two positive (respectively negative) functions, one has the following formulae.

Proposition 3.8. Let $f:\left(\mathbb{R}^{d_{1}}, 0\right) \longrightarrow(\mathbb{R}, 0)$ and $g:\left(\mathbb{R}^{d_{2}}, 0\right) \longrightarrow(\mathbb{R}, 0)$ be two positive or two negative real analytic function germs. Let us put $Z_{f}(T)=\sum_{n \geqslant 1} a_{n} T^{n}, Z_{g}(T)=\sum_{n \geqslant 1} b_{n} T^{n}$ and moreover $A_{n}=1-\sum_{j=1}^{n} a_{j}, B_{n}=1-\sum_{j=1}^{n} b_{j}$. Then the naive zeta function of $f * g:\left(\mathbb{R}^{d_{1}+d_{2}}, 0\right) \longrightarrow$ $(\mathbb{R}, 0)$ is $Z_{f * g}(T)=\sum_{n \geqslant 1} c_{n} T^{n}$, where

$$
c_{n}=a_{n} B_{n}+A_{n} b_{n}+a_{n} b_{n} .
$$

Example 3.9.

(i) Let $h: \mathbb{R}^{2} \longrightarrow \mathbb{R}$ be defined by $h(x, y)=x^{2}+y^{2}$. Recall that (cf. Example 3.4)

$$
Z_{f}(T)=\left(u^{2}-1\right) \sum_{n \geqslant 1} \frac{T^{2 n}}{u^{2 n}} .
$$

Putting $f(x)=g(x)=x^{2}$, then $h=f * g$ and by Example 3.1 we get that $a_{2 n}=b_{2 n}=$ $(u-1) / u^{n}$ and $a_{2 n+1}=b_{2 n+1}=0$; hence $A_{2 n}=A_{2 n+1}=1 / u^{n}$. Then, by Proposition 3.8, we rederive $c_{2 n}=\left(u^{2}-1\right) / u^{2 n}$ and $c_{2 n+1}=0$. 


\section{G. FICHOU}

(ii) Let $f$ and $g$ be defined by $f(x)=x^{2}$ and $g(y)=y^{4}$, and consider $f * g(x, y)=x^{2}+y^{4}$. The odd coefficients of the naive zeta function of $f * g$ are zero because $f$ and $g$ are positive, and it is easy to verify that

$$
a_{2 n}=\frac{u-1}{u^{n}}, A_{2 n}=\frac{1}{u^{n}} \quad \text { and } \quad b_{4 n}=\frac{u-1}{u^{n}}, b_{4 n+2}=0, B_{4 n}=\frac{1}{u^{n}}=B_{4 n+2} .
$$

Therefore $c_{4 n}=\left(u^{2}-1\right) / u^{3 n}$ and $c_{4 n+2}=(u-1) / u^{3 n+1}$, which was not so clear for the expression of the naive zeta function of $f * g$ computed with the Denef and Loeser formula, in Example 3.6(iii).

Proof of Proposition 3.8. We remark first that $u^{n d_{1}} A_{n}=\beta\left(\left\{\gamma \in \mathcal{L}_{n} ; \operatorname{ord}(f \circ \gamma)>n\right\}\right)$. In fact, the space $\mathcal{L}_{n}$ can be decomposed into the disjoint union

$$
\mathcal{L}_{n}=\pi_{n, 1}^{-1}\left(\mathcal{X}_{1}\right) \sqcup \ldots \sqcup \pi_{n, n}^{-1}\left(\mathcal{X}_{n}\right) \sqcup\left\{\gamma \in \mathcal{L}_{n} ; \operatorname{ord}(f \circ \gamma)>n\right\} .
$$

Hence, by additivity of $\beta$, one gets $u^{n d_{1}}=\sum_{j=1}^{n} a_{i} u^{n d_{1}}+\beta\left(\left\{\gamma \in \mathcal{L}_{n} ; \operatorname{ord}(f \circ \gamma)>n\right\}\right)$, and the remark is proved.

Now take $\left(\gamma_{1}, \gamma_{2}\right)$ in $\mathcal{L}_{n}\left(\mathbb{R}^{d_{1}}\right) \times \mathcal{L}_{n}\left(\mathbb{R}^{d_{2}}\right)=\mathcal{L}_{n}\left(\mathbb{R}^{d_{1}+d_{2}}\right)$. Then ord $\left(f \circ \gamma_{1}+g \circ \gamma_{2}\right)$ is greater than $n$ if and only if $\operatorname{ord}\left(f \circ \gamma_{1}\right)$ and $\operatorname{ord}\left(g \circ \gamma_{2}\right)$ are greater than $n$, because $f$ and $g$ are of the same sign. Therefore we have to distinguish the three cases:

(i) $\operatorname{ord}\left(f \circ \gamma_{1}\right)=n$ and $\operatorname{ord}\left(g \circ \gamma_{2}\right)>n$;

(ii) $\operatorname{ord}\left(f \circ \gamma_{1}\right)>n$ and $\operatorname{ord}\left(g \circ \gamma_{2}\right)=n$;

(iii) $\operatorname{ord}\left(f \circ \gamma_{1}\right)=n$ and $\operatorname{ord}\left(g \circ \gamma_{2}\right)=n$.

The computation gives

$$
\beta\left(\mathcal{X}_{n}(f * g)\right)=\beta\left(\mathcal{X}_{n}(f)\right) u^{n d_{2}} B_{n}+u^{n d_{1}} A_{n} \beta\left(\mathcal{X}_{n}(g)\right)+\beta\left(\mathcal{X}_{n}(f)\right) \beta\left(\mathcal{X}_{n}(g)\right) .
$$

\subsection{Motivic integration and the proof of the Denef and Loeser formula}

The proof of the Denef and Loeser formula, which is a simplification of that of [DL98, Theorem 2.2.1], to our setting, uses the theory of motivic integration on arc spaces for real algebraic varieties. In particular, we will use the change of variables formula of Kontsevich.

For the convenience of the reader, we recall briefly these notions before proving Propositions 3.2 and 3.5.

Take $\sigma:\left(M, \sigma^{-1}(0)\right) \longrightarrow\left(\mathbb{R}^{d}, 0\right)$ a real modification, and define the arc space associated to $\left(M, \sigma^{-1}(0)\right)$ by

$$
\mathcal{L}\left(M, \sigma^{-1}(0)\right)=\left\{\gamma:(\mathbb{R}, 0) \longrightarrow\left(M, \sigma^{-1}(0)\right) ; \gamma \text { is formal }\right\} .
$$

The truncated arc space $\mathcal{L}_{n}$ consists of the $\operatorname{arcs}$ of $\mathcal{L}$, but truncated at the order $n+1$, for an integer $n \geqslant 0$.

Denote by $\pi_{n}: \mathcal{L} \longrightarrow \mathcal{L}_{n}$ the natural truncation morphism, for $n \in \mathbb{N}$, where $\mathcal{L}$ denotes either $\mathcal{L}\left(M, \sigma^{-1}(0)\right)$ or $\mathcal{L}\left(\mathbb{R}^{d}, 0\right)$. A subset $A \subset \mathcal{L}$ is called stable if there exist a constructible set $C \subset \mathcal{L}_{n}$ and some $n \geqslant 0$ such that $A=\pi_{n}^{-1}(C)$. Then we can define the measure in $\mathbb{Z}\left[u, u^{-1}\right]$ of such a stable set $A$, with respect to the virtual Poincaré polynomial $\beta$, by

$$
\beta(A)=u^{-(n+1) d} \beta\left(\pi_{n}(A)\right),
$$

for $n$ large enough (note that $\beta\left(\pi_{n}(A)\right)$ is well-defined since Zariski constructible real algebraic varieties are arc-symmetric sets). Indeed, $\beta(A)$ does not depend on $n$ because the natural projections $\mathcal{L}_{n+1} \longrightarrow \mathcal{L}_{n}$ are locally trivial fibrations with fibre $\mathbb{R}^{d}$. 


\section{MotiviC INVARIANTS OF BLOW-NASH EQUIVALENCE}

Let us recall now the definition of integrals. Let $\theta: A \longrightarrow \mathbb{Z}\left[u, u^{-1}\right]$ be a map with a finite image and whose fibres are stable sets. Then the integral of $\theta$ over $A$ with respect to $\beta$ is defined by

$$
\int_{A} \theta d \beta=\sum_{c \in \mathbb{Z}\left[u, u^{-1}\right]} c \beta\left(\theta^{-1}(c)\right) .
$$

We can state the Kontsevich change of variables formula. Recall that jac $\sigma$ denotes the jacobian determinant of Jac $\sigma$.

Proposition 3.10 [Kon95, DL99]. Let $A \subset \mathcal{L}\left(\mathbb{R}^{d}, 0\right)$ be stable, and suppose that the function $\operatorname{ord}_{t} \operatorname{jac}_{\sigma}$ is bounded on $\sigma^{-1}(A)$. Then

$$
\beta(A)=\int_{\sigma^{-1}(A)} u^{-\operatorname{ord}_{t} \mathrm{jac} \sigma} d \beta
$$

Before giving the details of the proof of the Denef and Loeser formula, and notably a preliminary lemma, we fix some notations. Recall that $f$ is a Nash function germ. The modification $\sigma$ induces maps $\sigma_{*}$ (respectively $\sigma_{*, n}$ ) between $\mathcal{L}\left(M, \sigma^{-1}(0)\right)$ and $\mathcal{L}\left(\mathbb{R}^{d}, 0\right)$ (respectively $\mathcal{L}_{n}\left(M, \sigma^{-1}(0)\right.$ ) and $\left.\mathcal{L}_{n}\left(\mathbb{R}^{d}, 0\right)\right)$. Put

$$
\mathcal{Z}_{n}(f)=\pi_{n}^{-1}\left(\mathcal{X}_{n}\right) \quad \text { and } \quad \mathcal{Z}_{n}(f \circ \sigma)=\sigma_{*}^{-1}\left(\mathcal{Z}_{n}(f)\right) .
$$

Moreover, for $e \geqslant 1$, put $\Delta_{e}=\left\{\gamma \in \mathcal{L}\left(M, \sigma^{-1}(0)\right)\right.$; $\left.\operatorname{ord}_{t} \operatorname{jac} \sigma(\gamma(t))=e\right\}$, and finally define $\mathcal{Z}_{n, e}(f \circ \sigma)=\mathcal{Z}_{n}(f \circ \sigma) \cap \Delta_{e}$.

Lemma 3.11. Let $\sigma:\left(M, \sigma^{-1}(0)\right) \longrightarrow\left(\mathbb{R}^{d}, 0\right)$ be a modification of $\mathbb{R}^{d}$ such that $f \circ \sigma$ and the jacobian determinant jac $\sigma$ have only normal crossings simultaneously, and assume moreover that $\sigma$ is an isomorphism over the complement of the zero locus of $f$.

Let $(f \circ \sigma)^{-1}(0)=\bigcup_{j \in J} E_{j}$ be the decomposition of $(f \circ \sigma)^{-1}(0)$ into irreducible components, and assume that $\sigma^{-1}(0)=\bigcup_{k \in K} E_{k}$ for some $K \subset J$.

Put $N_{i}=\operatorname{mult}_{E_{i}} f \circ \sigma$ and $\nu_{i}=1+\operatorname{mult}_{E_{i}}$ jac $\sigma$, and for $I \subset J$ denote by $E_{I}^{0}$ the set $\left(\bigcap_{i \in I} E_{i}\right) \backslash\left(\bigcup_{j \in J \backslash I} E_{j}\right)$. Then there exists $c \in \mathbb{N}$ such that the naive zeta function $Z_{f}(T)$ of $f$ equals

$$
u^{d} \sum_{n \geqslant 1} T^{n} \sum_{e \leqslant c n} u^{-e} \sum_{I \neq \emptyset} \beta\left(\left\{\gamma \in \mathcal{L}_{n}\left(M, E_{I}^{0}\right) \cap \pi_{n}\left(\Delta_{e}\right) ; \text { ord } f \circ \sigma \circ \gamma=n\right\}\right)
$$

and the zeta functions with sign $Z_{f}^{ \pm}(T)$ equal

$$
u^{d} \sum_{n \geqslant 1} T^{n} \sum_{e \leqslant c n} u^{-e} \sum_{I \neq \emptyset} \beta\left(\left\{\gamma \in \mathcal{L}_{n}\left(M, E_{I}^{0}\right) \cap \pi_{n}\left(\Delta_{e}\right) ; f \circ \sigma \circ \gamma(t)= \pm t^{n}+\cdots\right\}\right) .
$$

Proof. Let us prove the lemma for $Z_{f}$ since the argument is the same for $Z_{f}^{ \pm}$.

For $n \geqslant 1, \mathcal{Z}_{n}(f)$ is stable, so $\beta\left(\mathcal{Z}_{n}(f)\right)$ is defined and equals $u^{-(n+1) d} \beta\left(\mathcal{X}_{n}\right)$, hence $Z_{f}(T)=$ $u^{d} \sum_{n \geqslant 1} \beta\left(\mathcal{Z}_{n}(f)\right) T^{n}$.

Moreover $\mathcal{Z}_{n}(f \circ \sigma)$ equals the disjoint union $\bigcup_{e \geqslant 1} \mathcal{Z}_{n, e}(f \circ \sigma)$, which is a finite union. Take $\gamma \in \mathcal{Z}_{n}(f \circ \sigma)$; there exists $I \subset J$ such that $\pi_{0}(\gamma) \in E_{I}^{0}$. Then in a neighbourhood of $\gamma(0)$, one can choose such coordinates that

$$
f \circ \sigma=\text { unit } \prod_{i \in I} y_{i}^{N_{i}} \quad \text { and } \quad \text { jac } \sigma=\text { unit } \prod_{i \in I} y_{i}^{\nu_{i}-1},
$$

where by unit we denote a non-vanishing analytic function. 


\section{G. FICHOU}

Let us write $\gamma=\left(\gamma_{1}, \ldots, \gamma_{d}\right)$ and $k_{i}=\operatorname{ord}_{t} \gamma_{i}$, for $i=1, \ldots, d$. Then the order $\operatorname{ord}_{t} f \circ \sigma(\gamma(t))$ is equal to $\sum_{i=1}^{d} N_{i} k_{i}=n$ and therefore

$$
\operatorname{ord}_{t} \operatorname{jac} \sigma(\gamma(t))=\sum_{i=1}^{d}\left(\nu_{i}-1\right) k_{i} \leqslant \max _{i}\left(\frac{\nu_{i}-1}{N_{i}}\right) \sum_{i=1}^{d} N_{i} k_{i}=\max _{i}\left(\frac{\nu_{i}-1}{N_{i}}\right) n \text {. }
$$

Let $c=\max _{i}\left(\left(\nu_{i}-1\right) / N_{i}\right)$. Then we have shown that $\bigcup_{e \geqslant 1} \mathcal{Z}_{n, e}(f \circ \sigma)=\bigcup_{e \leqslant c n} \mathcal{Z}_{n, e}(f \circ \sigma)$, where the union is finite.

Now the Kontsevich change of variables formula implies that $\beta\left(\mathcal{Z}_{n}(f)\right)$ is equal to the finite sum $\sum_{e \leqslant c n} u^{-e} \beta\left(\mathcal{Z}_{n, e}(f \circ \sigma)\right)$, and then $Z_{f}(T)=u^{d} \sum_{n \geqslant 1} T^{n} \sum_{e \leqslant c n} u^{-e} \beta\left(\mathcal{Z}_{n, e}(f \circ \sigma)\right)$.

We are going to compute $\beta\left(\mathcal{Z}_{n, e}(f \circ \sigma)\right)$ using the fact that $\mathcal{Z}_{n, e}(f \circ \sigma)$ equals the disjoint union $\bigsqcup_{I \neq \emptyset} \mathcal{Z}_{n, e}(f \circ \sigma) \cap \pi_{0}^{-1}\left(E_{I}^{0} \cap \sigma^{-1}(0)\right)$. Indeed, by additivity we find

$$
\beta\left(\mathcal{Z}_{n, e}(f \circ \sigma)\right)=\sum_{I \neq \emptyset} \beta\left(\mathcal{Z}_{n, e}(f \circ \sigma) \cap \pi_{0}^{-1}\left(E_{I}^{0} \cap \sigma^{-1}(0)\right)\right) .
$$

Choose $I \neq \emptyset$. Then $\pi_{n}\left(\mathcal{Z}_{n, e}(f \circ \sigma) \cap \pi_{0}^{-1}\left(E_{I}^{0} \cap \sigma^{-1}(0)\right)\right)$ is just the set

$$
\left\{\gamma(t) \in \mathcal{L}_{n}\left(M, \sigma^{-1}(0)\right) ; \gamma(0) \in E_{I}^{0} \cap \sigma^{-1}(0), \operatorname{ord}_{t} f \circ \sigma(\gamma)=n, \operatorname{ord}_{t} \operatorname{jac}(\gamma)=e\right\} .
$$

The result follows directly from the additivity of $\beta$.

The proof of Propositions 3.2 and 3.5 just consists in computing the value of the virtual Poincaré polynomial $\beta$ on the sets that appear in the formulae of Lemma 3.11.

Proof of Proposition 3.2. Take $\gamma \in \pi_{n}\left(\mathcal{Z}_{n, e}(f \circ \sigma) \cap \pi_{0}^{-1}\left(E_{I}^{0} \cap \sigma^{-1}(0)\right)\right)$. In a neighbourhood of $\gamma(0)$, one can choose such coordinates that

$$
f \circ \sigma=\text { unit } \prod_{i \in I} y_{i}^{N_{i}} \quad \text { and } \quad \operatorname{jac} \sigma=\text { unit } \prod_{i \in I} y_{i}^{\nu_{i}-1},
$$

hence $\pi_{n}\left(\mathcal{Z}_{n, e}(f \circ \sigma) \cap \pi_{0}^{-1}\left(E_{I}^{0} \cap \sigma^{-1}(0)\right)\right)$ is isomorphic to

$$
\left\{\gamma \in \mathcal{L}_{n}\left(M, \sigma^{-1}(0)\right) ; \gamma(0) \in E_{I}^{0} \cap \sigma^{-1}(0), \sum_{i \in I} k_{i} N_{i}=n, \sum_{i \in I} k_{i}\left(\nu_{i}-1\right)=e\right\},
$$

where $k_{i}=\operatorname{ord}_{t} \gamma_{i}$ for $i \in I$. As a consequence $\pi_{n}\left(\mathcal{Z}_{n, e}(f \circ \sigma) \cap \pi_{0}^{-1}\left(E_{I}^{0} \cap \sigma^{-1}(0)\right)\right)$ is isomorphic to

$$
\bigsqcup_{k \in A(n, e)}\left(E_{I}^{0} \cap \sigma^{-1}(0)\right) \times\left(\mathbb{R}^{*}\right)^{|I|}\left(\prod_{i \in I} \mathbb{R}^{n-k_{i}}\right) \times\left(\mathbb{R}^{n}\right)^{d-|I|},
$$

where $A(n, e)$ is the subset of $k \in \mathbb{N}^{d}$ defined by the equations $\sum_{i=1}^{d} N_{i} k_{i}=n$ and $\sum_{i=1}^{d}\left(\nu_{i}-1\right) k_{i}=e$.

By taking the image by $\beta$, we obtain the equality

$$
\beta\left(\pi_{n}\left(\mathcal{Z}_{n, e}(f \circ \sigma) \cap \pi_{0}^{-1}\left(E_{I}^{0} \cap \sigma^{-1}(0)\right)\right)\right)=\sum_{k \in A(n, e)} \beta\left(E_{I}^{0} \cap \sigma^{-1}(0)\right)(u-1)^{|I|} u^{n d-\sum_{i=1}^{d} k_{i}} .
$$

Hence the naive zeta function of $f$ satisfies

$$
Z_{f}(T)=\sum_{I \neq \emptyset}(u-1)^{|I|} \beta\left(E_{I}^{0} \cap \sigma^{-1}(0)\right) \sum_{n \geqslant 1} \sum_{e \leqslant c n} \sum_{k \in A(n, e)} u^{-e-\sum_{i=1}^{d} k_{i}} T^{n} .
$$

We remark that $\{k \in A(n, e) ; n \geqslant 1, e \leqslant c n\}$ is in bijection with $\mathbb{N}^{|I|}$; therefore

$$
\sum_{n \geqslant 1} \sum_{e \leqslant c n} \sum_{k \in A(n, e)} u^{-e-\sum_{i=1}^{d} k_{i}} T^{n}=\sum_{k} \prod_{i \in I}\left(u^{-\nu_{i}} T^{N_{i}}\right)^{k_{i}}=\prod_{i \in I} \frac{u^{-\nu_{i}} T^{N_{i}}}{1-u^{-\nu_{i}} T^{N_{i}}},
$$

and this ends the proof. 


\section{MotiviC INVARIANTS OF BLOW-NASH EQUIVALENCE}

The proof of Proposition 3.5 is a little bit more involved due to the fact that we have to introduce a covering $\widetilde{E_{I}^{0, \pm}}$ of $E_{I}^{0}$ in order to compute $Z_{f}^{ \pm}(T)$. Recall that if $U$ is an affine open subset of $M$ such that $f \circ \sigma=u \prod_{i \in I} y_{i}^{N_{i}}$ on $U$, where $u$ denotes a unit, then by $R_{U}^{ \pm}$we mean the set $R_{U}^{ \pm}=\left\{(x, t) \in\left(E_{I}^{0} \cap U\right) \times \mathbb{R} ; t^{m_{I}}= \pm 1 / u(x)\right\}$, where $m_{I}=\operatorname{gcd}_{i \in I}\left(N_{i}\right)$. Then $\widetilde{E_{I}^{0, \pm}}$ is the gluing of the $R_{U}^{ \pm}$along the $E_{I}^{0} \cap U$.

LEMma 3.12. The $R_{U}^{ \pm}$glue together along $E_{I}^{0} \cap U$.

Proof. It suffices to prove that the definition of $\widetilde{E_{I}^{0, \pm}}$ does not depend on the choice of the local coordinates. Let $z_{i}$ be another local system of coordinates on $U$ such that $f \circ \sigma=v \prod_{i \in I} z_{i}^{N_{i}}$. Then $z_{i}$ is proportional to $y_{i}$ for the indices $i$ in $I$; therefore $z_{i}=\alpha_{i} y_{i}$ for a non-vanishing analytic function $\alpha_{i}$. So $v(y) \prod_{i \in I} \alpha_{i}^{N_{i}}=u(y)$ and therefore the sets $\left\{(x, t) \in\left(E_{I}^{0} \cap U\right) \times \mathbb{R} ; t^{m_{I}}= \pm 1 / u(x)\right\}$ and $\left\{(x, t) \in\left(E_{I}^{0} \cap U\right) \times \mathbb{R} ; t^{m_{I}}= \pm 1 / v(x)\right\}$ are isomorphic by the map $(x, t) \longmapsto\left(x, t \prod_{i \in I} \alpha_{i}^{N_{i} / m_{I}}\right)$.

Proof of Proposition 3.5. Let $U$ be an affine open subset of $M$ such that $f \circ \sigma=u \prod_{i \in I} y_{i}^{N_{i}}$ on $U$, where $u$ is a unit. What we have to compute is the value of $\beta$ on

$$
W^{ \pm}=\left\{(x, y) \in\left(E_{I}^{0} \cap U\right) \times\left(\mathbb{R}^{*}\right)^{|I|} ; u(x) \prod_{i \in I} y_{i}^{N_{i}}= \pm 1\right\} .
$$

Denote by $m$ the greatest common divisor of the $N_{i}, i \in I$, and choose $n_{i}, i \in I$, such that $\sum_{i \in I} n_{i} N_{i}=m$. Assume that $I=\{1, \ldots, s\}$. We remark that $W^{ \pm}$is isomorphic to

$$
W^{\prime, \pm}=\left\{(x, y, t) \in\left(E_{I}^{0} \cap U\right) \times\left(\mathbb{R}^{*}\right)^{|I|} \times \mathbb{R}^{*} ; t^{m}=\frac{ \pm 1}{u(x)}, \prod_{i \in I} y_{i}^{N_{i} / m}=1\right\},
$$

by the map $(x, y, t) \longmapsto\left(x, t^{n_{1}} y_{1}, \ldots, t^{n_{s}} y_{s}\right)$ from $W^{\prime}, \pm$ to $W^{ \pm}$. The inverse is the morphism given by

$$
(x, y) \longmapsto\left(x,\left(\prod_{i \in I} y_{i}^{N_{i} / m}\right)^{-n_{1}} y_{1}, \ldots,\left(\prod_{i \in I} y_{i}^{N_{i} / m}\right)^{-n_{s}} y_{s}, \prod_{i \in I} y_{i}^{N_{i} / m}\right) .
$$

Now it is easier to compute $\beta\left(W^{\prime}, \pm\right)$ because $W^{\prime}, \pm \simeq R_{U}^{ \pm} \times\left(\mathbb{R}^{*}\right)^{|I|-1}$. This last isomorphism comes from the fact that at least one $N_{i} / m$ is odd. Therefore $\beta\left(W^{ \pm}\right)=(u-1)^{|I|-1} \beta\left(R_{U}^{ \pm}\right)$, and the same computation as in the naive case gives the formula.

\section{An invariant of the blow-Nash equivalence}

In this section, we define the blow-Nash equivalence of Nash function germs, by analogy with the blow-analytic equivalence due to Kuo [Kuo85]. He introduced this equivalence relation after noticing that the Whitney family, which is topologically trivial but has infinitely many $C^{1}$-equivalence classes, is analytically trivial after one blowing-up.

For a survey on blow-analytic equivalence, we refer to [FKK98]. Note in particular that this equivalence relation between real analytic function germs does not admit moduli for a family with isolated singularities. Moreover, there exist efficient tools to prove that two given germs are blow-analytically equivalent, for example via toric modifications. Note that there exist some recent invariants of this equivalence relation which enable one to distinguish blow-analytic types [Fuk97, KP03].

We prove that similar triviality results hold for this new equivalence relation. Moreover, we state and prove the main result of this section, which is the invariance of the zeta functions, constructed in $\S 3$, with respect to the blow-Nash equivalence. As an application, we finally state in $\S 4.2$ the blow-Nash equivalence classes of the two-variable Brieskorn polynomials. 


\section{G. FICHOU}

\subsection{Blow-Nash equivalence}

4.1.1 Definitions and remarks. To begin with, let us recall that by an algebraic modification of a real analytic function germ $f:\left(\mathbb{R}^{d}, 0\right) \longrightarrow(\mathbb{R}, 0)$, we mean a proper birational algebraic morphism $\sigma_{f}:\left(M_{f}, \sigma_{f}^{-1}(0)\right) \longrightarrow\left(\mathbb{R}^{d}, 0\right)$, between analytic neighbourhoods of 0 in $\mathbb{R}^{d}$ and the exceptional divisor $\sigma_{f}^{-1}(0)$ in $M_{f}$, which is an isomorphism over the complement of the zero locus of $f$ and for which $f \circ \sigma$ is in normal crossing.

DeFinition 4.1.

(i) A map $\phi:\left(\mathbb{R}^{d}, 0\right) \longrightarrow\left(\mathbb{R}^{d}, 0\right)$ is blow-Nash if there exists a proper birational algebraic morphism $\sigma:\left(M, \sigma^{-1}(0)\right) \longrightarrow\left(\mathbb{R}^{d}, 0\right)$ such that $\phi \circ \sigma$ is Nash (i.e. semi-algebraic and analytic).

(ii) Let $f, g:\left(\mathbb{R}^{d}, 0\right) \longrightarrow(\mathbb{R}, 0)$ be two germs of Nash functions. They are said to be blow-Nash equivalent if there exist two algebraic modifications

$$
\sigma_{f}:\left(M_{f}, \sigma_{f}^{-1}(0)\right) \longrightarrow\left(\mathbb{R}^{d}, 0\right) \text { and } \sigma_{g}:\left(M_{g}, \sigma_{g}^{-1}(0)\right) \longrightarrow\left(\mathbb{R}^{d}, 0\right),
$$

such that $f \circ \sigma_{f}$ and jac $\sigma_{f}$ (respectively $g \circ \sigma_{g}$ and jac $\sigma_{g}$ ) have only normal crossings simultaneously and a Nash isomorphism (i.e. a semi-algebraic map is an analytic isomorphism) $\Phi$ between analytic neighbourhoods $\left(M_{f}, \sigma_{f}^{-1}(0)\right)$ and $\left(M_{g}, \sigma_{g}^{-1}(0)\right)$ which preserves the multiplicities of the jacobian determinants of $\sigma_{f}$ and $\sigma_{g}$ along the components of the exceptional divisor, and which induces a homeomorphism $\phi:\left(\mathbb{R}^{d}, 0\right) \longrightarrow\left(\mathbb{R}^{d}, 0\right)$ such that $f=g \circ \phi$, as illustrated by the following commutative diagram.

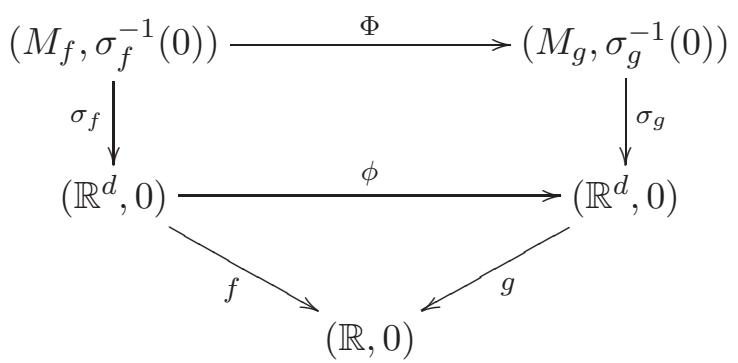

Remark 4.2 .

(i) We do not know whether the blow-Nash equivalence is an equivalence relation or not. When trying to adapt the corresponding proof for the blow-analytic equivalence [Kuo85], the problem comes from the transitivity property. Namely, the fibre product of an algebraic map and a Nash map need not be algebraic.

In the following, we consider the equivalence relation generated by this relation, which we still call blow-Nash equivalence.

(ii) Apart from the fact that the blow-Nash equivalence only concerns Nash germs, the differences between the blow-analytic equivalence (cf. [Kuo85]) and the blow-Nash equivalence are the following. In the definition of blow-Nash equivalence, we ask for the following:

(a) the modifications to be algebraic, and not only analytic, and their jacobian determinant to be in normal crossings,

(b) the isomorphism $\Phi$ upstairs to be Nash, and not only analytic,

(c) $\Phi$ to preserve the jacobian determinant orders of the modifications.

These additional assumptions are necessary in order to adapt techniques coming from motivic integration to this more analytic framework, and in particular to obtain the central result of this paper, Theorem 4.8. 


\section{Motivic InVARIANTS OF BLOW-NASH EQUivalence}

Note however that different definitions of a blow-analytic homeomorphism have occurred since the original article of Kuo [Kuo85] appeared, and notably Fukui, Kuo and Paunescu propose in [FKP01] a definition closer to ours. They define a blow-analytic isomorphism to be a homeomorphism such that there exists an analytic isomorphism upstairs which is moreover an isomorphism between the critical loci of the modifications.

(iii) Another relation close to blow-analytic equivalence, called blow-analytic equivalence, is also studied (cf. [FKK98]). It is defined in a similar way to blow-analytic equivalence, with the difference that the real analytic modifications are required to be compositions of blowings-up along smooth centres. Up to now, it is not known whether blow-analytic equivalence is an equivalence relation or not when $d>2$.

4.1.2 Properties. Here, we are concerned with triviality results and classification tools. First, it is obvious that two real analytic function germs that are analytically equivalent are blow-analytically equivalent! But note also that two Nash germs that are only analytically equivalent are also Nash equivalent, as proven by Shiota [Shi98], and therefore blow-Nash equivalent. Thus blow-Nash equivalence is a particular case of analytic equivalence between Nash germs.

Now, the question of moduli is a natural question when one studies an equivalence relation between germs. The following theorem states that there are no moduli for a Nash family with isolated singularities under some algebraicity assumptions on the modifications.

Theorem 4.3. Let $F:\left(\mathbb{R}^{d}, 0\right) \times P \longrightarrow(\mathbb{R}, 0)$ be Nash, where $P$ is a Nash set diffeomorphic to an open simplex in a euclidean space. Assume that $F(\cdot, p):\left(\mathbb{R}^{d}, 0\right) \longrightarrow \mathbb{R}$ has an isolated singularity at 0 for each $p \in P$, and assume moreover that $F$ admits an algebraic resolution of singularities.

Then the family $F(\cdot, p)$, for $p \in P$, consists of a finite number of blow-Nash equivalence classes.

Remark 4.4.

(i) By an algebraic resolution of singularities for $F$, we mean a finite composition $\beta: M \longrightarrow \mathbb{R}^{d} \times P$ of blowings-up with smooth algebraic centres such that $\beta$ is an isomorphism outside $0 \times P$, and $F \circ \beta$ is in normal crossing. Note that in the particular case where $F$ is polynomial, such a resolution exists by Hironaka's desingularization theorem [Hir64].

(ii) The proof of Theorem 4.3, which is postponed to $\S 4.3$, is inspired by [Kuo85] where Kuo proved the finiteness of the number of blow-analytic equivalence classes for an analytically parametrized family of isolated singularities. However, the key argument of integration along a vector field no longer applies in the Nash category, so we need new arguments.

The following particular case, which is a consequence of the proof of Theorem 4.3, gives a tool to prove blow-Nash triviality. It will help us in classifying the blow-Nash type of Brieskorn polynomials (see $\S 4.2)$.

COROllary 4.5. Let $f_{t}:\left(\mathbb{R}^{d}, 0\right) \longrightarrow(\mathbb{R}, 0), t \in I$, with $I$ an interval of $\mathbb{R}$, be a Nash parametrized family of weighted homogeneous polynomials of the same weight with an isolated singularity at the origin. Then the family $\left\{f_{t}\right\}_{t \in I}$ is blow-Nash trivial.

Proof. It is well known (see [FKK98, FP00] for example) that, in that case, one can find a toric modification $\beta:(M, E) \longrightarrow\left(\mathbb{R}^{d}, 0\right)$ of $\left(\mathbb{R}^{d}, 0\right)$, given by the weight of the weighted homogeneous polynomials, such that $\beta \times$ id fulfils the assumptions of Proposition 4.17.

Example 4.6. Consider the Nash function germs $x^{p}+y^{k p}$ and $x^{p}-y^{k p}$ from $\left(\mathbb{R}^{2}, 0\right)$ to $(\mathbb{R}, 0)$. They are blow-Nash equivalent. Indeed,

$$
f_{t}(x, y)=x^{p}+\frac{1-t^{2}}{1+t^{2}} p x y^{k(p-1)}+\frac{2 t}{1+t^{2}} y^{k p}, t \in[-1,1],
$$




\section{G. FICHOU}

is a weighted homogeneous polynomial of weight $(k, 1)$ with an isolated singularity at the origin for each $t \in[-1,1]$, and therefore Corollary 4.5 implies that $f_{-1}$ and $f_{1}$ are blow-Nash equivalent.

The counterpart is now to be able to distinguish blow-Nash types. Actually, it is a difficult issue to find invariants with this kind of relation. More precisely, for the blow-analytic equivalence, only the Fukui invariants [IKK02] and the zeta functions of Koike and Parusiński [KP03], defined with the Euler characteristic with compact supports, are known. Note that by evaluating the virtual Poincaré polynomial at $u=-1$ in the zeta functions defined in $\S 3$, one recovers these zeta functions. Moreover our zeta functions generalize the Fukui invariants. Indeed, it follows directly from Remark 2.11 that the following proposition is true.

Proposition 4.7. The Fukui invariants are the exponents of the naive zeta function $Z_{f}(T)$ with non-zero coefficients. Similarly, the Fukui invariants with sign are the exponents of the zeta functions with sign $Z_{f}^{ \pm}(T)$ with non-zero coefficients.

The following theorem is the main result of this paper.

THEOREM 4.8. The naive zeta function $Z_{f}(T)$ and the zeta functions with sign $Z_{f}^{ \pm}(T)$ of germs of Nash functions are invariants of the blow-Nash equivalence.

Proof. Let $f, g:\left(\mathbb{R}^{d}, 0\right) \longrightarrow(\mathbb{R}, 0)$ be two blow-Nash equivalent Nash function germs. By definition, there exist modifications

$$
\sigma_{f}:\left(M_{f}, \sigma_{f}^{-1}(0)\right) \longrightarrow\left(\mathbb{R}^{d}, 0\right) \quad \text { and } \quad \sigma_{g}:\left(M_{g}, \sigma_{g}^{-1}(0)\right) \longrightarrow\left(\mathbb{R}^{d}, 0\right),
$$

and a Nash isomorphism $\Phi:\left(M_{f}, \sigma_{f}^{-1}(0)\right) \longrightarrow\left(M_{g}, \sigma_{g}^{-1}(0)\right)$ as in Definition 4.1. Then the assumptions of Propositions 3.2 and 3.5 are satisfied.

Now, it suffices to prove that the expressions of the zeta functions given by the Denef and Loeser formulae coincide. But $\beta$ is invariant under Nash isomorphisms by Theorem 2.16, and moreover $\Phi$ preserves

(a) the multiplicities of $f \circ \sigma_{f}$ and $g \circ \sigma_{g}$, because it is an isomorphism,

(b) the multiplicities of the jacobians of $\sigma_{f}$ and $\sigma_{g}$ along the components of the exceptional divisors, by definition of blow-Nash equivalence.

Therefore the zeta functions of $f$ and $g$ coincide.

Remark 4.9 .

(i) If the zeta functions generalize the Fukui invariants and the zeta functions defined with the Euler characteristic with compact supports, they are invariants for a more restrictive relation between germs.

(ii) However, our zeta functions are also invariants for the analytic equivalence of real analytic germs. In fact, the constructible sets $\mathcal{X}_{n}$ and $\mathcal{X}_{n}^{ \pm}$associated with two analytically equivalent function germs $f, g$ are isomorphic. Indeed, let $h$ be a local analytic isomorphism such that $f=$ $g \circ h$. Then, the map $\gamma \longmapsto \pi_{n}(h(\gamma))$ from $\mathcal{X}_{n}(f)$ to $\mathcal{X}_{n}(g)$ is an algebraic isomorphism because, after truncation at the level of the space of arcs, the local analytic isomorphism becomes algebraic. Therefore the naive zeta functions of $f$ and $g$ coincide. The proof in the case with sign is similar.

\subsection{Application to Brieskorn polynomials}

We apply our zeta functions to sketch the classification of two-variable Brieskorn polynomials under blow-Nash equivalence, and to give examples in three variables. Brieskorn polynomials in two or 


\section{MotiviC INVARIANTS OF BLOW-NASH EQUIVALENCE}

three variables are polynomials of the type

$$
\varepsilon_{p} x^{p}+\varepsilon_{q} y^{q}\left(+\varepsilon_{r} z^{r}\right), p \leqslant q \leqslant r \in \mathbb{N}, \varepsilon_{p}, \varepsilon_{q}, \varepsilon_{r} \in\{ \pm 1\} .
$$

We remark that, if $p=1$, then $\varepsilon_{p} x+\varepsilon_{q} y^{q}\left(+\varepsilon_{r} z^{r}\right)$ is Nash isomorphic to $x$. Therefore we will restrict our attention to the case $p \geqslant 2$.

The classification under blow-analytic equivalence has been done completely in the two-variable case, and almost completely in the three-variable case in [KP03] using the zeta functions defined with Euler characteristic with compact supports and the Fukui invariants (see [KP03, Theorem 7.3]; for the Fukui invariants, see [IKK02]). There exists only one case that cannot be decided, and the following example shows that we can distinguish it under blow-Nash equivalence. However, this is not sufficient to come to any conclusion in the blow-analytic context.

Example 4.10. Let $f_{p, k}$ be the Brieskorn polynomial defined by $f_{p, k}= \pm\left(x^{p}+y^{k p}+z^{k p}\right)$, with $p$ even, $k \in \mathbb{N}$. We prove that for fixed $p$ and different $k$, two such polynomials are not blow-Nash equivalent.

In order to do this, we calculate directly the naive zeta function of $f_{p, k}$. For $n \in \mathbb{N}$, we have to compute $\beta\left(\mathcal{X}_{n}\right)$. First, it is clear that $\mathcal{X}_{n}=\emptyset$ when $n$ is not a multiple of $p$. If $n$ is a multiple of $p$, write $n=p(m k+r)$ where $m k+r$ represents the euclidean division of $n / p$ by $k$. If $\gamma \in \mathcal{L}_{n}$, put $\gamma=\left(a_{1} t+\cdots+a_{n} t^{n}, b_{1} t+\cdots+b_{n} t^{n}, c_{1} t+\cdots+c_{n} t^{n}\right)$.

Then if $r \neq 0$, the first non-zero term of $f \circ \gamma$ is given by the first component of $\gamma$; hence $\mathcal{X}_{n}$ equals

$$
\left\{\gamma ; a_{m k+r} \neq 0, a_{1}=\cdots=a_{m k+r-1}=b_{1}=\cdots=b_{m}=c_{1}=\cdots=c_{m}=0\right\} .
$$

In the case where $r=0$, the three components of $\gamma$ play a part, and $\mathcal{X}_{n}$ equals

$$
\left\{\gamma ;\left(a_{m k}, b_{m}, c_{m}\right) \neq 0, a_{1}=\cdots=a_{m k-1}=b_{1}=\cdots=b_{m-1}=c_{1}=\cdots=c_{m-1}=0\right\} .
$$

Therefore

$$
\mathcal{X}_{n} \simeq \begin{cases}\mathbb{R}^{*} \times \mathbb{R}^{(p-1)(m k+r)} \times\left(\mathbb{R}^{p(m k+r)-m}\right)^{2} & \text { if } r \neq 0, \\ \left(\mathbb{R}^{3}\right)^{*} \times \mathbb{R}^{(p-1) m k} \times\left(\mathbb{R}^{p m k-m}\right)^{2} & \text { if } r=0,\end{cases}
$$

and hence the coefficient of $T^{n}$ is

$$
\beta\left(\mathcal{X}_{n}\right) u^{-3 n}= \begin{cases}(u-1) u^{-(m k+r)-2 m} & \text { if } n=p(m k+r), 0<r<k, \\ \left(u^{3}-1\right) u^{-m k-2 m} & \text { if } n=p m k, \\ 0 & \text { otherwise. }\end{cases}
$$

Therefore the zeta function of $f_{p, k}$ looks like

$$
\begin{aligned}
Z_{f_{p, k}}= & (u-1)\left(u^{-1} T^{p}+u^{-2} T^{2 p}+\cdots+u^{-(k-1)} T^{(k-1) p}\right)+\left(u^{3}-1\right) u^{-k-2} T^{k p} \\
& +(u-1)\left(u^{-(k+3)} T^{(k+1) p}+u^{-(k+4)} T^{(k+2) p}+\cdots+u^{-(2 k+1)} T^{(2 k-1) p}\right) \\
& +\left(u^{3}-1\right) u^{-2(k-2)} T^{2 k p}+\cdots
\end{aligned}
$$

Now it suffices to note that, for $p$ fixed and $k<k^{\prime}$, the $p k$ coefficient of $Z_{f_{p, k}}$ is $\left(u^{3}-1\right) u^{-k-2}$ whereas that of $Z_{f_{p, k^{\prime}}}$ is $(u-1) u^{-k}$.

Remark 4.11. The case of two-variable Brieskorn polynomials has been dealt with in [KP03], using their zeta functions and the Fukui invariants. The only case where the equivalence class of Brieskorn polynomials of two variables cannot be distinguished using only their zeta functions, and which requires the use of the Fukui invariants, is the following: $f_{k}(x, y)= \pm\left(x^{k}+y^{k}\right), k \geqslant 2$ even. We remark that we have seen in Example 3.4 that for $k \neq k^{\prime}$ the naive zeta functions $Z_{f_{k}}$ and $Z_{f_{k}^{\prime}}$ are different; therefore our zeta function distinguishes this case, with respect to the blow-Nash equivalence. 


\section{G. FICHOU}

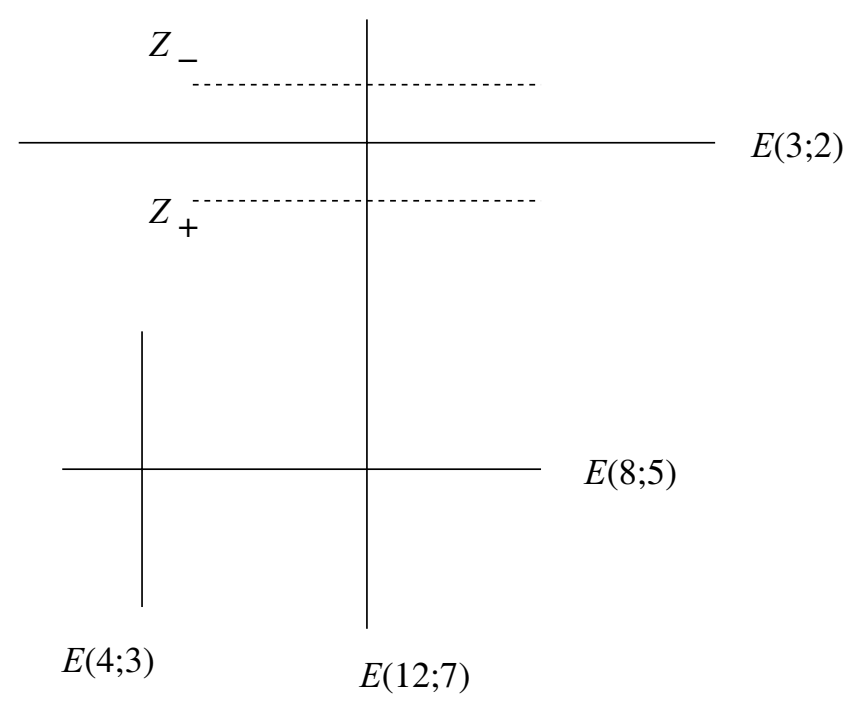

Figure 1. Resolution tree of $x^{3} \pm y^{4}$.

The fact, one can say more. Indeed, for two-variable Brieskorn polynomials the naive zeta function determines the exponents $p$ and $q$.

Proposition 4.12. Let $g= \pm x^{p} \pm y^{q}$ be a two-variable Brieskorn polynomial. Then the exponents $p$ and $q$ are uniquely determined by the naive zeta function $Z_{g}(T)=\sum_{n \geqslant 1} g_{n} T^{n}$. More precisely

$$
p=\min \left\{n ; g_{n} \neq 0\right\},
$$

and if $l=\min \left\{n ; g_{n} \neq a_{n} u^{n}\right\}$, where $\sum_{n \geqslant 1} a_{n} T^{n}$ denotes the naive zeta function of $\pm x^{p}$, then

$$
q= \begin{cases}l-1 & \text { if } p \text { is odd, } p \text { divides } l-1 \text { and } g_{l} \neq(u-1) u^{k} \text { for any } k \in \mathbb{N}, \\ l & \text { otherwise. }\end{cases}
$$

Proof. The characterization of $p$ is clear. Now, if $p \nmid q$, then $q=l$ and $g_{q}=(u-1) u^{k}$ for some $k \in \mathbb{N}$.

If $q=k p$ for some $k \in \mathbb{N}$, then $g_{k p}=\beta\left(\left\{ \pm a^{p} \pm b^{k p}\right\}\right) u^{2 k p-k-1}$ and thus

$$
g_{k p}=a_{k p} u^{k p} \Longleftrightarrow \beta\left(\left\{ \pm a^{p} \pm b^{k p} \neq 0\right\}\right)=u(u-1) \Longleftrightarrow p \text { odd. }
$$

In that case $g_{k p+1}=(u-1)^{2} u^{2 k p-k-2}$, so $l=k p+1$.

Therefore, if $p$ is even, then $q=l$; and if $p$ is odd, then either $q=l$ or $q=l-1$. More precisely, if $g_{l}=(u-1) u^{k}$, then $p \nmid q$ and $q=l$; whereas if $g_{l}=(u-1)^{2} u^{k}$, then $p \mid l-1$ and $q=l-1$.

To find the signs in front of $x^{p}$ and $y^{q}$, the naive zeta function is not sufficient, as illustrated by the following example.

Example 4.13. Let $f_{ \pm}:\left(\mathbb{R}^{2}, 0\right) \longrightarrow(\mathbb{R}, 0)$ be defined by $f_{ \pm}(x, y)=x^{3} \pm y^{4}$. One can resolve the singularities of $f_{ \pm}$by a succession of four blowings-up. The resolution tree of this modification $\sigma$ is drawn in Figure 1 , where $Z_{ \pm}$denotes the strict transform of $f_{ \pm}$, and $E(N ; \nu)$ denotes the irreducible component of the exceptional divisor such that mult $f_{ \pm} \circ \sigma=N$ and $1+$ mult $_{E}$ jac $\sigma=\nu$. The Denef and Loeser formulae imply that $Z_{f_{+}}$is equal to $Z_{f_{-}}$, but $f_{+}$and $f_{-}$are not blow-Nash equivalent (they are not even blow-analytically equivalent; see [KP03, Theorem 6.1], for example).

The zeta functions with sign enable one to discuss the signs for two-variable Brieskorn polynomials except in one case. The following proposition specifies the possibilities. Note that 


\section{MotiviC INVARIANTS OF BLOW-NASH EQUIVALENCE}

$(x, y) \longrightarrow( \pm x, \pm y)$ gives an action on the blow-Nash classes; hence when the power $p$ (or $q$ ) is odd, the corresponding sign cannot be determined. By convention, in the case where $p=q$ and the signs are opposite, we consider $x^{p}-y^{p}$ rather than $-x^{p}+y^{p}$.

Proposition 4.14. Let $Z_{g}^{ \pm}(T)=\sum_{n \geqslant 1} g_{n}^{ \pm} T^{n}$ be the zeta functions with sign of $\varepsilon_{p} x^{p}+\varepsilon_{q} y^{q}$, with $\varepsilon_{p}, \varepsilon_{q} \in\{ \pm 1\}$. If $p$ is even, then

$$
\varepsilon_{p}= \begin{cases}+1 & \text { if } g_{p}^{+} \neq 0 \\ -1 & \text { otherwise }\end{cases}
$$

and if $q$ is even, but not a multiple of an odd $p$, then

$$
\varepsilon_{q}= \begin{cases}+1 & \text { if } g_{p}^{-}=0 \\ -1 & \text { otherwise }\end{cases}
$$

The remaining case is when $p$ is odd and $q=k p$ with $k$ even. But in example 4.6 we proved that in that case $x^{p}+y^{k p}$ and $x^{p}-y^{k p}$ are blow-Nash equivalent, and therefore the following proposition holds.

Proposition 4.15. For two Brieskorn polynomials in two variables, the following three statements are equivalent:

(i) they are blow-Nash equivalent,

(ii) they are blow-analytically equivalent,

(iii) their naive zeta function and zeta functions with sign coincide.

\subsection{Proof of Theorem 4.3}

Kuo [Kuo85] proved the finiteness of the number of blow-analytic equivalence classes for an analytically parametrized family of real analytic germs with an isolated singularity. In that setting, he used integration along vector fields to construct a trivialization of a modification of the zero set of the family. Unfortunately, in the Nash situation, this efficient method is forbidden, for we go outside of the Nash world.

Fortunately Fukui, Koike and Shiota have given an effective tool to show Nash triviality: the Nash isotopy lemma [FKS98]. It gives a trivialization of Nash submanifolds, possibly with boundary, with normal crossings, and also of their arbitrary intersections.

Theorem 4.16 (Nash isotopy lemma). Let $M$ be a Nash manifold possibly with boundary and $N_{1}, \ldots, N_{k}$ be Nash submanifolds of $M$ possibly with boundary which together with $N_{0}=\partial M$ are normal crossing. Assume that $\partial N_{i} \subset N_{0}$ for $i \in\{1, \ldots, k\}$. Let $P$ be a Nash manifold diffeomorphic to an open simplex in a euclidean space, and $\omega: M \longrightarrow P$ be a proper onto Nash submersion such that the restrictions of $\omega$,

$$
\omega: N_{i_{1}} \cap \cdots \cap N_{i_{s}} \longrightarrow P,
$$

for $0 \leqslant i_{1}<\cdots<i_{s} \leqslant k$, are also proper onto submersions.

Then there exists a Nash isomorphism

$$
\phi:\left(M ; N_{1}, \ldots, N_{k}\right) \longrightarrow\left(M \cap \omega^{-1}(0) ; N_{1} \cap \omega^{-1}(0), \ldots, N_{k} \cap \omega^{-1}(0)\right) \times P
$$

such that $\omega \circ \phi^{-1}:\left(M \cap \omega^{-1}(0)\right) \times P \longrightarrow P$ is the canonical projection.

This result does not totally replace integration along vector fields because it works just at the level of manifolds, and not of functions. Therefore it enables us to obtain a Nash trivialization of the zero set of our Nash parametrized family of Nash germs, but not of the non-zero levels of the functions of the family. Now, in order to show the Nash triviality, we have recourse here to orthogonal 


\section{G. FICHOU}

projections between levels of functions (cf. Lemma 4.18) in order to force the trivialization. The point is that, if this technique does not allow us to keep a Nash isomorphism, it gives us a blow-Nash isomorphism, which is sufficient for the case we are considering.

Proof of Theorem 4.3. Following Kuo's proof [Kuo85], we can subdivide $P$ into a finite number of Nash sets $P^{\prime}$, diffeomorphic to open simplices in a euclidean space, such that there exists an algebraic modification $\beta: M \longrightarrow \mathbb{R}^{d} \times P^{\prime}$ which satisfies

(a) $\beta$ is an isomorphism outside $0 \times P^{\prime}$,

(b) $F \circ \beta$ is in normal crossing,

(c) if $r: \mathbb{R}^{d} \times P^{\prime} \longrightarrow P^{\prime}$ denotes the canonical projection, then $r \circ \beta$ maps the canonical strata of $(F \circ \beta)^{-1}(0)$ submersively onto $P^{\prime}$.

Now the Nash isotopy lemma enables us to apply Proposition 4.17 below, and thus to prove that two germs $F(\cdot, p)$ and $F(\cdot, q)$ with $p, q \in P^{\prime}$ are blow-Nash equivalent.

So, thanks to the Nash isotopy lemma, we are led to study the particular case where the family $F:\left(\mathbb{R}^{d}, 0\right) \times P \longrightarrow \mathbb{R}$ admits, as a resolution of singularities, a product $\beta \times \operatorname{id}_{P}$, where $\beta:(M, E) \longrightarrow$ $\left(\mathbb{R}^{d}, 0\right)$ is a proper birational morphism, which is an isomorphism outside 0 , and $F \circ\left(\beta \times \operatorname{id}_{P}\right)$ has only normal crossings. More precisely, we mean that, in that case, there exist local systems of parameters $\left(x_{1}, \ldots, x_{d}\right)$ centred at a point in $E \times P$ such that

$$
F \circ\left(\beta \times \operatorname{id}_{P}\right)\left(x_{1}, \ldots, x_{d}, p\right)=u_{p}(x) \prod_{i=1}^{d} x_{i}^{r_{i}},
$$

where $u_{p}$ is a non-vanishing Nash function.

In that setting, we have the following triviality result.

Proposition 4.17. Let $F:\left(\mathbb{R}^{d}, 0\right) \times P \longrightarrow \mathbb{R}$ be a Nash mapping, where $P$ is a connected Nash manifold, and assume that $f_{p}=F(\cdot, p):\left(\mathbb{R}^{d}, 0\right) \longrightarrow \mathbb{R}$ has an isolated singularity at 0 for each $p \in P$. Assume moreover that there exists a proper birational morphism $\beta:(M, E) \longrightarrow\left(\mathbb{R}^{d}, 0\right)$, with exceptional divisor $E$, such that the product $\beta \times \operatorname{id}_{P}$ is a resolution for $F$. Then the family $F(\cdot, p)$ consists of a unique blow-Nash equivalence class.

Proof. We prove Proposition 4.17 in several steps. By assumption we dispose of a trivialization (the identity map of $M$ ) of the zero sets of $F(\cdot, p)$ for $p \in P$. But there is no chance that this trivialization respects the levels of $F(\cdot, p)$. Actually we are going to project the trivialization of the zero level in order to force it also to trivialize the other levels.

Note that $M$, as a modification of an affine space, is affine, and so one can assume $M \subset \mathbb{R}^{N}$. Denote $d=\operatorname{dim} M$.

We show first that we can define locally the projection directly on $M$, without blowing-up. For simplicity, denote $F(\cdot, p)$ by $F_{p}$.

Lemma 4.18. Take $x \in M$ and $p_{0} \in P$. Then there exists $\eta_{x}>0$ such that the orthogonal projection $\phi(x, p)$ of $x$ onto the level $\left\{F_{p}=F_{p_{0}}(x)\right\}$ is well defined for $\left|p-p_{0}\right|<\eta_{x}$.

Proof. If $x \in E$, then put $\phi(x, p)=x$. Now, if $x \notin E$, then $F_{p}(x) \neq 0$ for all $p$ in $P$. But for $c \neq 0$, there exists a Nash tubular neighbourhood (cf. [BCR98, Corollary 8.9.5]) of the level $\left\{F_{p_{0}}=c\right\}$. Therefore, for $p$ sufficiently close to $p_{0}, x$ belongs to the Nash tubular neighbourhood of $\left\{F_{p_{0}}=\right.$ $\left.F_{p}(x)\right\}$, and then $\phi(x, p)$ is defined as the unique orthogonal projection of $x$ onto $\left\{F_{p_{0}}=F_{p}(x)\right\}$. 


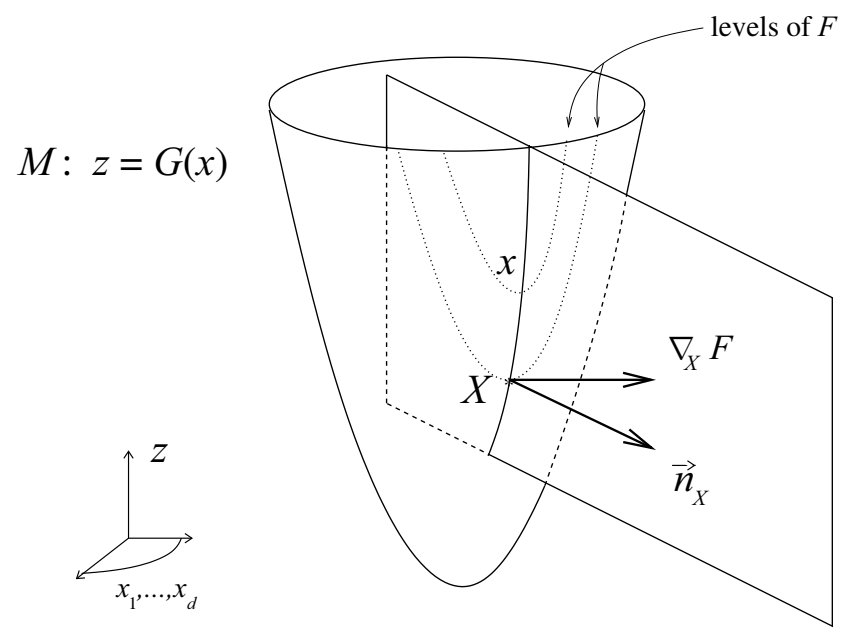

Figure 2. Local situation.

Remark 4.19. Such a construction does not seem to be so easy to perform in a global situation because the width of the Nash tubular neighbourhood $\left\{F_{p_{0}}=c\right\}$, for a fixed function $F_{p_{0}}$, tends to 0 as $c$ tends to 0 . Therefore it does not seem to be reasonable to hope for a global strictly positive $\eta$.

Now let us perform the computation of the projection in the local case as illustrated in Figure 2. To simplify the notation, we assume in the following that $M$ is a hypersurface of $\mathbb{R}^{N}$, that is $N=d+1$. Take $x_{0} \in E \subset M \subset \mathbb{R}^{N}$, and $p_{0} \in P$. For simplicity assume also that $p_{0}=0$. There exist a system of parameters centred at $x_{0}$ such that:

(i) $M$ is locally the graph $z=G\left(x_{1}, \ldots, x_{d}\right)$ of a Nash function $G$,

(ii) if we extend $F \circ\left(\beta \times \operatorname{id}_{P}\right)$ to a function on $\mathbb{R}^{N}$ in the trivial way, then $F \circ\left(\beta \times \operatorname{id}_{P}\right)$ is of the form

$$
F \circ\left(\beta \times \operatorname{id}_{P}\right)(x, z, p)=u_{p}(x) \prod_{i=1}^{d} f_{i}(x)^{r_{i}},
$$

where $u_{p}$ is a Nash function in $(x, p)$ which does not vanish and $f_{1}, \ldots, f_{d}$ are normal crossings functions.

For $(x, p) \in M \times P$, the orthogonal projection $\phi(x, p)=X$ satisfies

$$
\begin{aligned}
x & =X+c \nabla_{X} F-k \nabla_{X} G, \\
z & =Z+k, \\
F(x, z, 0) & =F(X, Z, p),
\end{aligned}
$$

where $\nabla_{X} G$ (respectively $\nabla_{X} F$ ) denotes the gradient vector of $G$ (respectively $F$ ) at $X$.

Lemma 4.20. The orthogonal projection $\phi$ is a blow-Nash isomorphism from a neighbourhood of $(0,0)$ in $\mathbb{R}^{d} \times P$ to a neighbourhood of $(0,0)$ in $\mathbb{R}^{d} \times P$.

Proof. Let us prove the result in two steps. As a first step we prove that $\phi$ is blow-Nash, and afterwards we show that $\phi$ is a blow-Nash isomorphism.

First step. In order to prove the lemma, it suffices to show that the map

$$
(X, p) \stackrel{\psi}{\longrightarrow}\left(X+c(X, p) \nabla_{X} F-k(X, p) \nabla_{X} G, p\right),
$$




\section{G. FICHOU}

which is by (1) the inverse of $\phi$, is a blow-Nash isomorphism between two neighbourhoods of $(0,0)$ in $\mathbb{R}^{d} \times P$. As $c$ and $k$ are given by implicit formulae, we want to apply the implicit function theorem, which makes sense in the Nash setting (cf. [BCR98]). However, the computation downstairs shows us that we cannot apply this theorem directly, and we need to separate the divisors given by $f_{i}=0$ by several blowings-up, for each $i$ such that $r_{i} \neq 0$.

To begin with, let us specify the members of the last equation of system (1) in coordinates. The left one is

$$
F(x, z, 0)=u_{0}\left(X+c \nabla_{X} F-k \nabla_{X} G\right) \prod_{i=1}^{d} f_{i}\left(X+c \nabla_{X} F-k \nabla_{X} G\right)^{r_{i}}
$$

and the right one is

$$
F(X, Z, p)=u_{p}(X) \prod_{i=1}^{d} f_{i}(X)^{r_{i}}
$$

We remark that the Taylor formula applied to $f_{i}$ implies that

$$
f_{i}(X+H)=f_{i}(X)+\left\langle\nabla_{X} f_{i}, H\right\rangle+h_{i, X}(H),
$$

where $h_{i}$ is a Nash function such that $\left\|h_{i, X}(H)\right\| \leqslant K\|H\|^{2}$ in some neighbourhood of 0 , for some positive constant $K$.

Therefore $f_{i}\left(X+c \nabla_{X} F-k \nabla_{X} G\right)$ is equal to

$$
f_{i}(X)+c\left\langle\nabla_{X} f_{i}, \nabla_{X} F\right\rangle-k\left\langle\nabla_{X} f_{i}, \nabla_{X} G\right\rangle+h_{i, X}\left(c \nabla_{X} F-k \nabla_{X} G\right) .
$$

Assume now, for simplicity, that the number of divisors $f_{i}=0$ is equal to $d$ (that is, $r_{i}>0$ for all $i$ ). We separate these divisors $f_{i}=0$ by a succession of $d$ blowings-up with respect to ideal sheaves corresponding to blowing up the origin, then the total transform of one-dimensional intersections, etc. By symmetry, it suffices to perform the computation in one chart, and therefore one can assume this modification $\pi$ to be given in the chart $\mathcal{U}$ by

$$
\left(f_{1}, f_{2}, \ldots, f_{d}\right)=\left(Y_{1}, Y_{1} Y_{2}, \ldots, Y_{1} Y_{2} \cdots Y_{d}\right)
$$

Let us denote by $c^{\prime}$ and $k^{\prime}$ the modified forms of $c$ and $k$ respectively. Put

$$
e_{i}=\sum_{j=i}^{d} r_{j}, \quad \widetilde{c}(Y, p)=\left(\prod_{i=1}^{d} Y_{i}^{e_{i}-2}\right) c^{\prime}, \quad \widetilde{k}(Y, p)=\frac{k^{\prime}}{\left(\prod_{i=1}^{d} Y_{i}\right) \widetilde{c}(Y, p)}
$$

and

$$
V=\sum_{i=1}^{d} r_{i}\left(\prod_{j=i+1}^{d} Y_{j}\right) \nabla_{\pi(Y)} f_{i}
$$

We remark that $V$ cannot vanish since the $f_{i}$ form a system of coordinates.

Then, after the modification, the term $c \nabla_{X} F$ is transformed into

$$
u_{p} \widetilde{c}\left(\prod_{i=1}^{d} Y_{i}\right) V+\widetilde{c}\left(\prod_{i=1}^{d} Y_{i}\right)^{2} \nabla_{\pi(Y)} u_{p}
$$




\section{MotiviC INVARIANTS OF BLOW-NASH EQUIVALENCE}

As a consequence $f_{i}\left(X+c \nabla_{X} F-k \nabla_{X} G\right)$ becomes $Y_{1} \cdots Y_{i}+\widetilde{c}\left(\prod_{i=1}^{d} Y_{i}\right) \Lambda_{i}$, where

$$
\begin{aligned}
\Lambda_{i}= & u_{p}\left\langle\nabla_{\pi(Y)} f_{i}, V\right\rangle+\widetilde{c}\left(\prod_{i=1}^{d} Y_{i}\right)\left\langle\nabla_{\pi(Y)} f_{i}, \nabla_{\pi(Y)} u_{p}\right\rangle-\widetilde{k}\left\langle\nabla_{\pi(Y)} f_{i}, \nabla_{\pi(Y)} G\right\rangle \\
& +\frac{h_{i, X}\left(\widetilde{c}\left(\prod_{i=1}^{d} Y_{i}\right)\left(V+\left(\prod_{i=1}^{d} Y_{i}\right) \nabla_{\pi(Y)} u_{p}-\widetilde{k} \nabla_{\pi(Y)} G\right)\right)}{\widetilde{c}\left(\prod_{i=1}^{d} Y_{i}\right)} .
\end{aligned}
$$

Therefore equation (2) is transformed into

$$
\sum_{l=1}^{r} \widetilde{c}^{l} \sum_{\substack{j_{1}+\ldots+j_{d}=l \\
j_{i} \in\left\{1, \ldots, r_{i}\right\}}} \prod_{i=1}^{d}\left(\begin{array}{c}
j_{i} \\
r_{i}
\end{array}\right) \Lambda_{i}^{j_{i}} Y_{i}^{j_{1}+\cdots+j_{i-1}}=v(\widetilde{c}, \widetilde{k}, Y)
$$

with

$$
v(\widetilde{c}, \widetilde{k}, Y)=\frac{u_{p}(\pi(Y))}{u_{0}\left(\pi(Y)+\widetilde{c}\left(\prod_{i=1}^{d} Y_{i}\right)\left(V-\widetilde{k} \nabla_{\pi(Y)} G\right)\right)}-1 .
$$

So $\widetilde{c}$ and $\widetilde{k}$ are given implicitly by

$$
\begin{aligned}
& E_{1}\left(\widetilde{c}, \widetilde{k}, Y_{1}, p\right)=\sum_{l=1}^{r} \widetilde{c}^{l} \sum_{\substack{j_{1}+\cdots+j_{d}=l \\
j_{i} \in\left\{1, \ldots, r_{i}\right\}}} \prod_{i=1}^{d}\left(\begin{array}{c}
j_{i} \\
r_{i}
\end{array}\right) \Lambda_{i}^{j_{i}} Y_{i}^{j_{1}+\cdots+j_{i-1}}-v(\widetilde{c}, \widetilde{k}, Y)=0, \\
& E_{2}\left(\widetilde{c}, \widetilde{k}, Y_{1}, p\right)=\widetilde{k}-\left(G\left(\pi(Y)+\left(\prod_{i=1}^{d} Y_{i}\right) \widetilde{c}\left(V-\widetilde{k} \nabla_{\pi(Y)} G\right)\right)-G \circ \pi(Y)\right)=0,
\end{aligned}
$$

where we consider $Y_{2}, \ldots, Y_{d}$ as parameters. Now we can apply the implicit function theorem. First, remark that $E_{1}(0,0,0,0)=0$ and $E_{2}(0,0,0,0)=0$. Now, let us make explicit the coefficient of $\widetilde{c}$ in equation (4). This coefficient is just $\sum_{i=1}^{d} r_{i}\left(\prod_{j=i+1}^{d} Y_{j}\right) \Lambda_{i}-a$, where $a$ is the contribution coming from $-v(\widetilde{c}, \widetilde{k}, Y)$. Thus the coefficient of $\widetilde{c}$ equals

$$
\begin{aligned}
u_{p}\|V\|^{2} & +\left(\prod_{i=1}^{d} Y_{i}\right)\left\langle V, \nabla_{\pi(Y)} u_{p}\right\rangle-\widetilde{k}\left\langle V, \nabla_{\pi(Y)} G\right\rangle \\
& +\frac{\sum_{i=1}^{d} r_{i}\left(\prod_{j=i+1}^{d} Y_{j}\right) h_{i, X}\left(\widetilde{c}\left(\prod_{i=1}^{d} Y_{i}\right)\left(V+\left(\prod_{i=1}^{d} Y_{i}\right) \nabla_{\pi(Y)} u_{p}-\widetilde{k} \nabla_{\pi(Y)} G\right)\right)}{\widetilde{c}\left(\prod_{i=1}^{d} Y_{i}\right)}-a .
\end{aligned}
$$

Note that $a$ tends to zero as $\left(Y_{1}, p\right)$ tends to $(0,0)$.

Now, it is easy to compute the jacobian matrix of $\left(E_{1}, E_{2}\right)$ with respect to the variables $(\widetilde{c}, \widetilde{k})$ at the point $(0,0,0,0)$. The result is

$$
\left(\begin{array}{ll}
\frac{\partial E_{1}}{\partial \widetilde{c}}(0,0,0,0) & \frac{\partial E_{1}}{\partial \widetilde{k}}(0,0,0,0) \\
\frac{\partial E_{2}}{\partial \widetilde{c}}(0,0,0,0) & \frac{\partial E_{2}}{\partial \widetilde{k}}(0,0,0,0)
\end{array}\right)=\left(\begin{array}{cc}
u_{p}(0)\|V\|^{2} & 0 \\
0 & 1+\left\|\nabla_{\pi(Y)} G\right\|^{2}
\end{array}\right)
$$

which is an invertible matrix because $u_{p}$ and $V$ do not vanish. Therefore $\widetilde{c}$ and $\widetilde{k}$ are defined and blow-Nash in a neighbourhood of $(0,0)$ in $M \times P$.

Now, let us come back to $\psi$. For a fixed $p$, write $\psi_{p}(\cdot)$ instead of $\psi(\cdot, p)$. Then $\psi_{p}$ is defined in a neighbourhood $\mathcal{V}\left(\mathbb{R}^{d}\right)$ of 0 in $\mathbb{R}^{d}$, and in restriction to a neighbourhood of $Y_{1}=0$ in the chart $\mathcal{U}$, 


\section{G. FICHOU}

one has

$$
\psi_{p} \circ \pi(Y)=\left(Y_{1}+\widetilde{c}\left(\prod_{i=1}^{d} Y_{i}\right) W_{1}, Y_{1} Y_{2}+\widetilde{c}\left(\prod_{i=1}^{d} Y_{i}\right) W_{2}, \ldots, Y_{1} Y_{2} \cdots Y_{d}+\widetilde{c}\left(\prod_{i=1}^{d} Y_{i}\right) W_{d}\right),
$$

where $W$ is the vector $W=V-\widetilde{k} \nabla_{\pi(Y)} G$.

Then $\psi_{p}$ lifts to a function $\widetilde{\psi}_{p}$

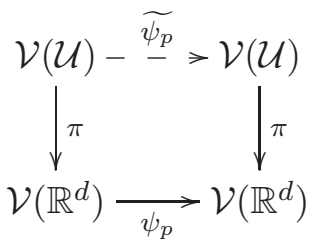

between neighbourhoods of $Y_{1}=0$ in $\mathcal{U}$, with $\widetilde{\psi}_{p}$ being given in coordinates by

$$
\widetilde{\psi}_{p}(Y)=\left(Y_{1}\left(1+\widetilde{c}\left(\prod_{i=2}^{d} Y_{i}\right) W_{1}\right), Y_{2} \frac{1+\widetilde{c}\left(\prod_{i=3}^{d} Y_{i}\right) W_{2}}{1+\widetilde{c}\left(\prod_{i=2}^{d} Y_{i}\right) W_{1}}, \ldots, Y_{d} \frac{1+\widetilde{c} W_{d}}{1+\widetilde{c} Y_{d} W_{d-1}}\right) .
$$

Note that the denominators cannot vanish in a neighbourhood of $\left(Y_{1}, p\right)=(0,0)$ because $\widetilde{c}$ is small for $\left(Y_{1}, p\right)$ sufficiently small.

Second step. It suffices to prove that the jacobian determinant of $\widetilde{\psi}$ is non-zero for $Y_{1}$ and $p$ small because $\widetilde{\psi}$ is a bijection. Indeed, $\widetilde{\psi}$ is a bijection outside $E$ because so is $\psi$, and restricted to $E$, it is just the identity.

Note that $\widetilde{c}$ and $\partial \widetilde{c} / \partial Y_{i}$, for $i \in\{2, \ldots, d\}$, vanish when $\left(Y_{1}, p\right)=(0,0)$. Therefore, evaluated at $\left(Y_{1}, p\right)=(0,0)$,

$$
\frac{\partial \widetilde{\psi}^{i}}{\partial Y_{i}}=1, \quad \frac{\partial \widetilde{\psi}^{i}}{\partial Y_{j}}=0
$$

if $i \neq j$ and $j \neq 1$. So the jacobian determinant of $\widetilde{\psi}$ equals 1 at $\left(Y_{1}, p\right)=(0,0)$ and thus is non-zero for $Y_{1}$ and $p$ small.

Remark 4.21. The constructed projection is locally the minimal one because it is trivial if $x \in E$ or $p=0$.

Now, we have to prove that these projections glue together.

Lemma 4.22. The projections of Lemma 4.20 glue together.

Proof. Cover $E$ by a finite number of neighbourhoods of the kind in Lemma 4.20 using the compactness of $E$. We can assume that the intersections two by two of these neighbourhoods are connected. Then, we dispose of a finite number of projections $\phi^{i}$ defined in neighbourhoods of the form $V^{i} \times B\left(p_{0}, \eta_{i}\right)$, with $\eta_{i}>0$. Denote by $\eta$ the minimal of the rays $\eta_{i}$ and by $U$ the union of the neighbourhoods. We are going to prove that the projections $\phi^{i}$ glue together on $U \times B\left(p_{0}, \eta\right)$.

Assume that there exists a point $x$ in the intersection $V_{1} \cap V_{2}$. Then, in a neighbourhood of $\left(x, p_{0}\right)$ in $M \times P$, the three projections, $\phi^{1}, \phi^{2}$ and that of Lemma 4.18 , coincide by minimality. Therefore the analytic functions $\phi^{1}$ and $\phi^{2}$, which coincide on a non-isolated set of points, are equal on the connected set $V_{1} \cap V_{2}$.

The proof of Proposition 4.17 now follows easily from these lemmas. In fact, it suffices to prove that two germs $F_{p}$ and $F_{q}$ are blow-Nash equivalent for $p$ and $q$ sufficiently close to each other, and the lemmas give a relevant blow-Nash isomorphism in that case. 


\section{MotiviC INVARIANTS OF BLOW-NASH EQUIVALENCE}

\section{ACKNOWLEDGEMENT}

I am greatly indebted to Adam Parusiński, my thesis advisor at the University of Angers, where this work was carried out, for his help during this work.

\section{REFERENCES}

AKMW02 D. Abramovich, K. Karu, K. Matsuki and J. Wlodarczyk, Torification and factorization of birational maps, J. Amer. Math. Soc. 15 (2002), 531-572.

BM97 E. Bierstone and P. D. Milman, Canonical desingularization in characteristic zero by blowing up the maximum strata of a local invariant, Invent. Math. 128 (1997), 207-302.

Bit04 F. Bittner, The universal Euler characteristic for varieties of characteristic zero, Compositio Math. 140 (2004), 1011-1032.

BCR98 J. Bochnak, M. Coste and M.-F. Roy, Real algebraic geometry (Springer, Berlin, 1998).

DL98 J. Denef and F. Loeser, Motivic Igusa zeta functions, J. Algebraic Geom. 7 (1998), 505-537.

DL99 J. Denef and F. Loeser, Germs of arcs on singular algebraic varieties and motivic integration, Invent. Math. 135 (1999), 201-232.

DL01 J. Denef and F. Loeser, Geometry on arc spaces of algebraic varieties, in European congress of mathematics, Barcelona, 2000, Progress in Mathematics, vol. 201 (Birkhäuser, Basel, 2001), $327-348$.

DL02 J. Denef and F. Loeser, Lefschetz numbers of iterates of the monodromy and truncated arcs, Topology 41 (2002), 1031-1040.

FKK98 T. Fukui, S. Koike and T.-C. Kuo, Blow-analytic equisingularities, properties, problems and progress, in Real analytic and algebraic singularities, eds T. Fukuda, T. Fukui, S. Izumiya and S. Koike, Pitman Research Notes in Mathematics Series, vol. 381 (Pitman, London, 1998), 8-29.

FKP01 T. Fukui, T.-C. Kuo and L. Paunescu, Constructing blow-analytic isomorphisms, Ann. Inst. Fourier (Grenoble) $\mathbf{5 1}$ (2001), 1071-1087.

FKS98 T. Fukui, S. Koike and M. Shiota, Modified Nash triviality of a family of zero-sets of real polynomial mappings, Ann. Inst. Fourier (Grenoble) 48 (1998), 1395-1440.

FP00 T. Fukui and L. Paunescu, Modified analytic trivialization for weighted homogeneous functiongerms, J. Math. Soc. Japan 52 (2000), 433-446.

Fuk97 T. Fukui, Seeking invariants for blow-analytic equivalence, Compositio Math. 105 (1997), 95-107.

Ful93 W. Fulton, Introduction to toric varieties, Annals of Mathematics Studies, vol. 131 (Princeton University Press, Princeton, NJ, 1993).

Hir64 H. Hironaka, Resolution of singularities of an algebraic variety over a field of characteristic zero, Ann. of Math. (2) 79 (1964), 109-326.

IKK02 S. Izumi, S. Koike and T.-C. Kuo, Computations and stability of the Fukui invariant, Compositio Math. 130 (2002), 49-73.

Koi97 S. Koike, Modified Nash triviality theorem for a family of zero-sets of weighted homogeneous polynomial mappings, J. Math. Soc. Japan 49 (1997), 617-631.

Koi00 S. Koike, Nash trivial simultaneous resolution for a family of zero-sets of Nash mappings, Math. Z. 234 (2000), 313-338.

Kon95 M. Kontsevich, Lecture at Orsay, 7 December 1995.

KP03 S. Koike and A. Parusiński, Motivic-type invariants of blow-analytic equivalence, Ann. Inst. Fourier (Grenoble) $\mathbf{5 3}$ (2003), 2061-2104.

Kuo85 T.-C. Kuo, On classification of real singularities, Invent. Math. 82 (1985), 257-262.

Kur88 K. Kurdyka, Ensembles semi-algébriques symétriques par arcs, Math. Ann. 282 (1988), 445-462.

Kur99 K. Kurdyka, Injective endomorphisms of real algebraic sets are surjective, Math. Ann. 313 (1999), 69-82. 


\section{Motivic INVARIANTS OF BLOW-NASH EQUIVALENCE}

Loj91 S. Lojasiewicz, Introduction to complex analytic geometry (Birkhäuser, Basel, 1991).

Loo02 E. Looijenga, Motivic measures, in Séminaire Bourbaki, exposé 874, Astérisque 276 (2002), 267-297.

MCP03 C. McCrory and A. Parusiński, Virtual Betti numbers of real algebraic varieties, C. R. Math. Acad. Sci. Paris 336 (2003), 763-768.

Par04 A. Parusiński, Topology of injective endomorphisms of real algebraic sets, Math. Ann. 328 (2004), 353-372.

Qua01 R. Quarez, Espace des germes d'arcs réels et série de Poincaré d'un ensemble semi-algébrique, Ann. Inst. Fourier (Grenoble) 51 (2001), 43-68.

Shi98 M. Shiota, Relation between equivalence relations of maps and functions, in Real analytic and algebraic singularities, eds T. Fukuda, T. Fukui, S. Izumiya and S. Koike, Pitman Research Notes in Mathematics Series, vol. 381 (Pitman, London, 1998), 114-144.

Tot02 B. Totaro, Topology of singular algebraic varieties, in Proc. Int. Cong. Mathematicians, Beijing, 2002, vol. 2 (Higher Ed. Press, Beijing, 2002), 533-541.

Wlo03 J. Wlodarczyk, Toroidal varieties and the weak factorization theorem, Invent. Math. 154 (2003), $223-331$.

Goulwen Fichou goulwen.fichou@univ-rennes1.fr

IRMAR, Université de Rennes 1, Campus Beaulieu, F-35042 Rennes cedex, France 